\title{
Finite Difference Simulations of Seismic Scattering: Implications for the Propagation of Short-Period Seismic Waves in the Crust and Models of Crustal Heterogeneity
}

\author{
Arthur Frankel ${ }^{1}$ \\ Jet Propulsion Laboratory, California Institute of Technology, Pasadena \\ Robert W. Clayton \\ Seismological Laboratory, California Institute of Technology, Pasadena
}

\begin{abstract}
Synthetic seismograms produced by the finite difference method are used to study the scattering of elastic and acoustic waves in two-dimensional media with random spatial variations in seismic velocity. The results of this study provide important insights about the propagation of short-period $(<1 \mathrm{~s})$ seismic waves in the earth's crust and place significant constraints on the fluctuation spectrum of crustal heterogeneity on length scales from tens of kilometers to tens of meters. The synthetic seismograms are analyzed to determine the variation in travel times and waveforms across arrays of receivers. The apparent attenuation caused by scattering and the time decay and amplitude of the seismic coda are also quantified with the numerical simulations. Random media with Gaussian and exponential correlation functions are considered, as well as a self-similar medium with equal variations in seismic velocity over a broad range of length scales. These media differ in the spectral falloff of their velocity fluctuations at wavelengths smaller than $2 \pi$ times the correlation distance $a$. The synthetic seismograms demonstrate that a random medium with self-similar velocity fluctuations at length scales less than about $50 \mathrm{~km}$ $(a \geq 10 \mathrm{~km})$ can explain both travel time anomalies reported for teleseismic arrivals across large-scale seismic arrays (e.g., LASA and NORSAR) and the presence of seismic coda at frequencies of $30 \mathrm{~Hz}$ and greater commonly observed in microearthquake waveforms. Media with Gaussian and exponential correlation functions in velocity do not account for both sets of observations for reasonable standard deviations in velocity $(\leq 10 \%)$. The scattering attenuation $\left(Q^{-1}\right)$ observed in the simulations for Gaussian media is peaked at $k a$ between 1 and 2 , where $k$ is the seismic wave number. The observed attenuation in exponential media increases with frequency for $k a<1$ and remains about constant for $1 \leq k a \leq 5.6$. At high frequencies ( $k a>5$ ), the self-similar medium is characterized by a scattering $Q$ that is constant with frequency, whereas theory predicts that the apparent $Q$ in an exponential medium is proportional to frequency. These alternative models of crustal heterogeneity can thus be tested by improved measurements of the frequency dependence of crustal $Q$ at frequencies greater than about $1 \mathrm{~Hz}$, assuming that scattering is responsible for most of the attenuation at these frequencies. Measurements of the time decay of the synthetic coda waves clearly show that the single scattering model of coda decay is not appropriate in the presence of moderate amounts of scattering attenuation (scattering $Q \leq 200$ ). In these cases, $Q$ values derived from the coda decay rate using the single scattering theory do not correspond to the transmission $Q$ of the medium. The cross correlation of synthetic waveforms observed for an array of receivers along the free surface is observed to be dependent on the correlation distance of the medium. The self-similar random medium proposed here for the crust produces waveform variations at high frequencies $(15-30 \mathrm{~Hz})$ similar to those reported for actual small-scale seismic arrays with apertures of hundreds of meters.
\end{abstract}

\section{INTRODUCTION}

The scattering of short-period seismic waves $(T<1 \mathrm{~s})$ by heterogeneities in the earth's crust and upper mantle affects a number of seismic observables, including amplitudes, travel times, spectra, and waveforms. A full understanding of seismic scattering is clearly necessary to comprehend the propagation of short-period seismic waves in the lithosphere. We use the term "scattering" in this paper to refer to the interaction of seismic waves with spatial variations in material properties of the medium, variations that range in size from several seismic wavelengths to a small fraction of a wavelength. The study of short-period seismic waves is of increasing interest to seismologists, as more attention is devoted to the evaluation of high-

\footnotetext{
${ }^{1}$ Now at U.S. Geological Survey, Menlo Park, California.

Copyright 1986 by the American Geophysical Union.
}

Paper number 5B5778.

0148-0227/86/005B-5778\$05.00 frequency strong ground motions, the estimation of earthquake and explosion source parameters from high-frequency data, and the analysis of high-resolution seismic exploration surveys.

In this paper, we are concerned with variations in seismic velocity in the earth's crust with scale lengths ranging from tens of kilometers down to tens of meters. Relatively largescale variations $(>10 \mathrm{~km})$ in crustal velocity have been identified in tomographic inversions of travel time anomalies observed at seismic arrays for both teleseismic waves [Aki et al., 1977] and local earthquakes (see, e.g., Hearn and Clayton $[1986 a, b])$. Scattering from smaller-scale heterogeneities $(<1$ $\mathrm{km}$ across) represents one possible mechanism for the generation of seismic coda that is generally observed for microearthquake waveforms at frequencies greater than $1 \mathrm{~Hz}$ [Aki, 1969]. It has been proposed that scattering from such small-scale heterogeneities is responsible for the apparent attenuation of seismic waves in the lithosphere at frequencies from 1 to $25 \mathrm{~Hz}[A k i, 1980 a, b]$. Theoretical and numerical studies demonstrate that scattering produces apparent attenu- 

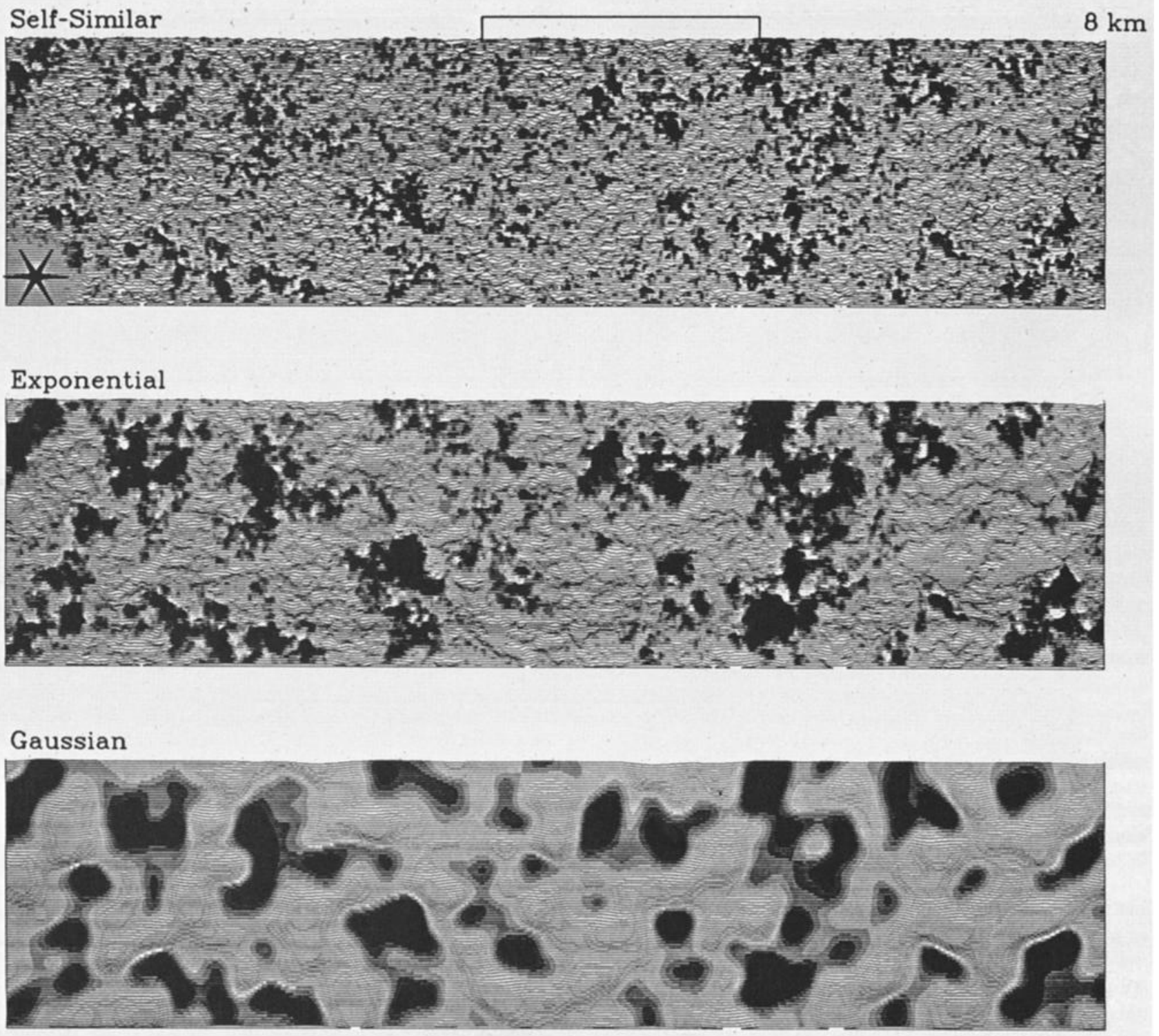

Fig. 1. Representations of the three types of random media considered in this study. Each medium shown has a correlation distance of $200 \mathrm{~m}$. The amplitude of each horizontal line denotes the random component of the $P$ or $S$ wave velocity $\left(V_{P} / V_{S}\right.$ constant). Areas with higher than average velocity are shaded. In the top panel, the positions of the explosion source (star) and receiver array (bar) along the free surface are shown. This configuration was used in the simulations described in the section on high-frequency coda amplitude and cross correlation.

ation with distance in a manner similar, in some respects, to intrinsic loss mechanisms [Wu, 1982; Richards and Menke, 1983; Frankel and Clayton, 1984; Malin and Phinney, 1985]. Scattering also causes variations in waveforms and amplitudes across seismic arrays [see Ringdal and Husebye, 1982].

We seek to explain these various manifestations of scattering with a single random medium model of the heterogeneity of the crust. Such a model should account for observations over a broad spectrum of seismic frequencies $(1-30 \mathrm{~Hz})$ and describe the spectrum of velocity fluctuations in the crust over a wide range of length scales (tens of meters to tens of kilometers).

We use synthetic seismograms generated by the finite difference method to study waves propagating through twodimensional random media. These random media consist of a small component ( $\leq 10 \%$ standard deviation) of their $P$ and $S$ wave velocities varying randomly in space, superimposed on some average velocities. Such media are meant to approximate the heterogeneity of the crust caused by variations in composition and the presence of fractures and fluid-filled inclusions. Examples of the three types of random media considered in this paper are shown in Figure 1. Each medium is described by its spatial autocorrelation function, which is commonly referred to as simply the "correlation function." The media vary in their spectrum of fluctuations and will be discussed in detail in the following section.

\section{Finite Difference Method APPlied TO RANDOM MEDIA}

The finite difference method propagates complete seismic wave fields through a two-dimensional grid with arbitrarily complex variations in material properties. The application of this technique to seismic wave propagation in random media 
TABLE 1. Correlation Functions and Spectra for Random Media Studied

\begin{tabular}{lccc}
\hline \multicolumn{1}{c}{ Correlation Function } & $N(r)$ & $\begin{array}{c}\text { One-Dimensional } \\
\text { Fourier Transform }\end{array}$ & $\begin{array}{c}\text { Two-Dimensional } \\
\text { Fourier Transform }\end{array}$ \\
\hline Gaussian & $e^{-r^{2} / a^{2}}$ & $(\pi)^{1 / 2} a e^{-k^{2} a^{2} / 4}$ & $\frac{a^{2}}{2} e^{-k_{r} 2 a^{2} / 4}$ \\
Exponential & $e^{-r / a}$ & $\frac{2 a}{1+k^{2} a^{2}}$ & $\frac{a^{2}}{\left(1+k_{r}^{2} a^{2}\right)^{3 / 2}}$ \\
Von Karman: self-similar* & $K_{0}\left(\frac{r}{a}\right)$ & $\frac{a}{\left(1+k^{2} a^{2}\right)^{1 / 2}}$ & $\frac{a^{2}}{1+k_{r}^{2} a^{2}}$ \\
\hline
\end{tabular}

*See Appendix B for normalization.

was first described by Frankel and Clayton [1984]. The finite difference method has several advantages over theoretical treatments of scattering. It produces synthetic seismograms for any point on the grid. It includes all multiply scattered waves, converted waves ( $P$ to $S, S$ to $P$ ), diffractions, and caustics. Most theoretical studies of seismic scattering assume the first Born approximation, which states that only single scattering occurs and that scattering losses from the primary wave can be neglected [Pekeris, 1947; Chernov, 1960]. This approximation is valid only for weakly scattering media and appears inappropriate for many portions of the crust [Hudson and Heritage, 1981; Richards and Menke, 1983]. Unlike some approaches to scattering which assume the ray approximation, the finite difference method is not restricted to certain ratios of wavelength to scatterer size and can be used for media with velocity fluctuations over a range of length scales.

The two-dimensional acoustic wave equation describing pressure $(P)$ in a medium where the velocity $v$ is a function of $x$ and $z$ and the density is constant is given by

$$
P_{, t t}=v^{2}(x, z)\left(P_{, x x}+P_{, z z}\right)
$$

Here subscripts after commas indicate partial derivatives. For elastic waves, the two coupled equations for the horizontal $(u)$ and vertical $(w)$ components of displacement are

$$
\begin{aligned}
& \rho u_{. t t}=\left[(\lambda+2 \mu) u_{. x}+\lambda w_{, z}\right]_{, x}+\left[\mu\left(w_{, x}+u_{, z}\right)\right]_{, z} \\
& \rho w_{, t t}=\left[(\lambda+2 \mu) w_{, z}+\lambda u_{, x}\right]_{, z}+\left[\mu\left(w_{, x}+u_{, z}\right)\right]_{, x}
\end{aligned}
$$

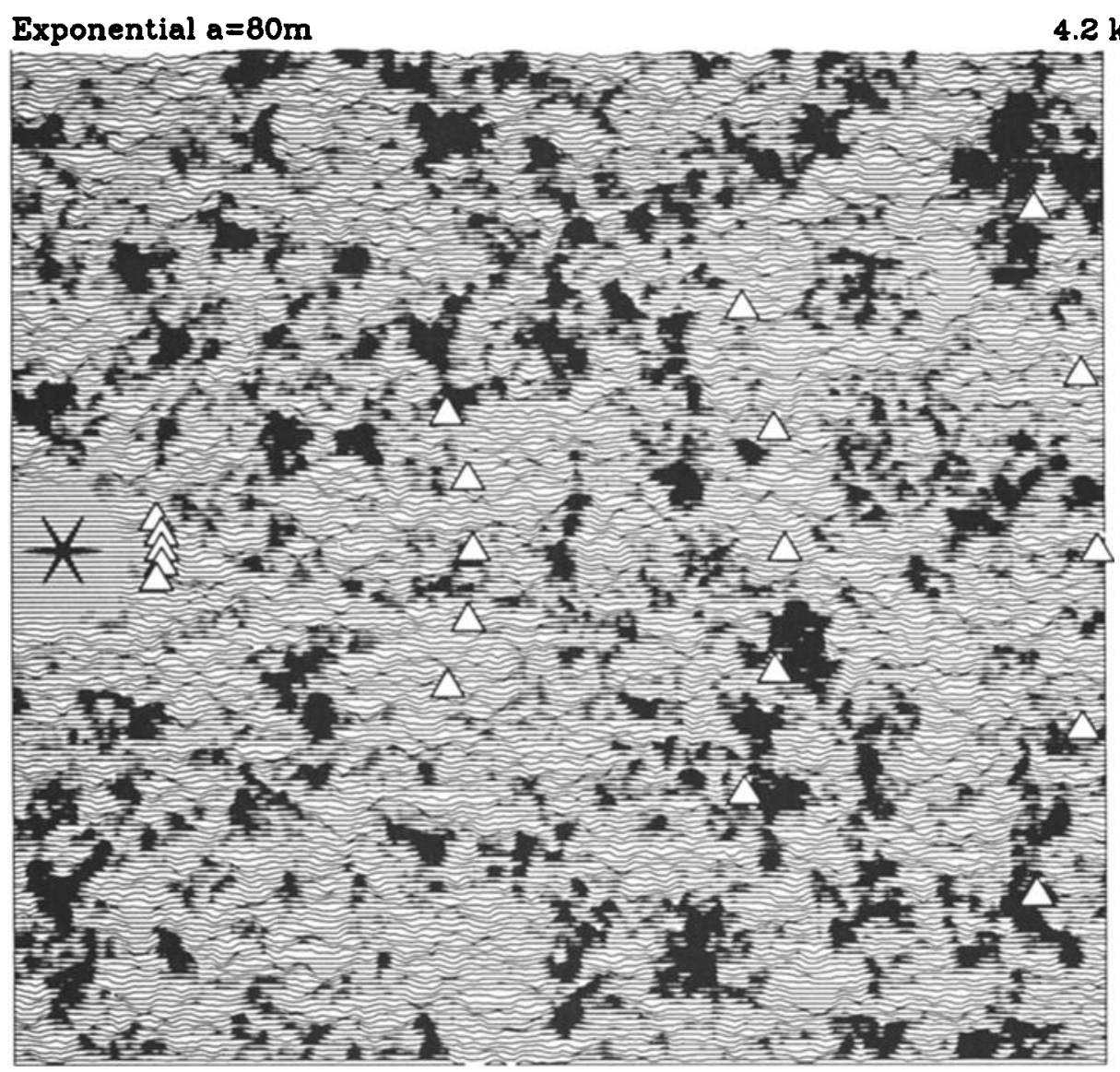

Fig. 2. Typical configuration used for the study of apparent attenuation. The star denotes the location of the explosion source, and the triangles are the receivers. All edges have absorbing boundary conditions. The grid size is 210 by 200 points, with a grid spacing of $20 \mathrm{~m}$. The medium has an exponential correlation function and a correlation distance of 80 m. 


\section{P-Wave (Divergence)}
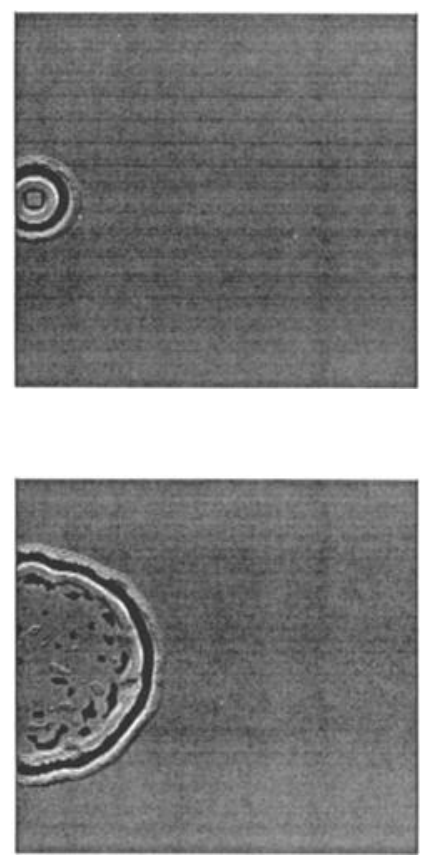

$.192 \mathrm{sec}$
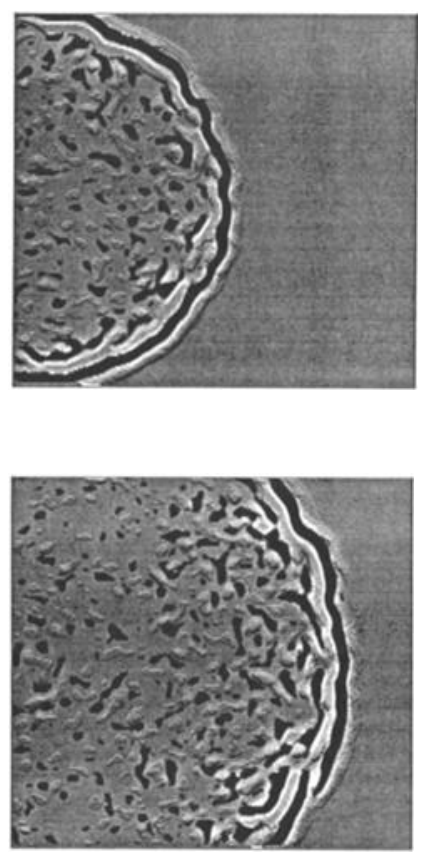

$.320 \mathrm{sec}$

$.064 \mathrm{sec}$
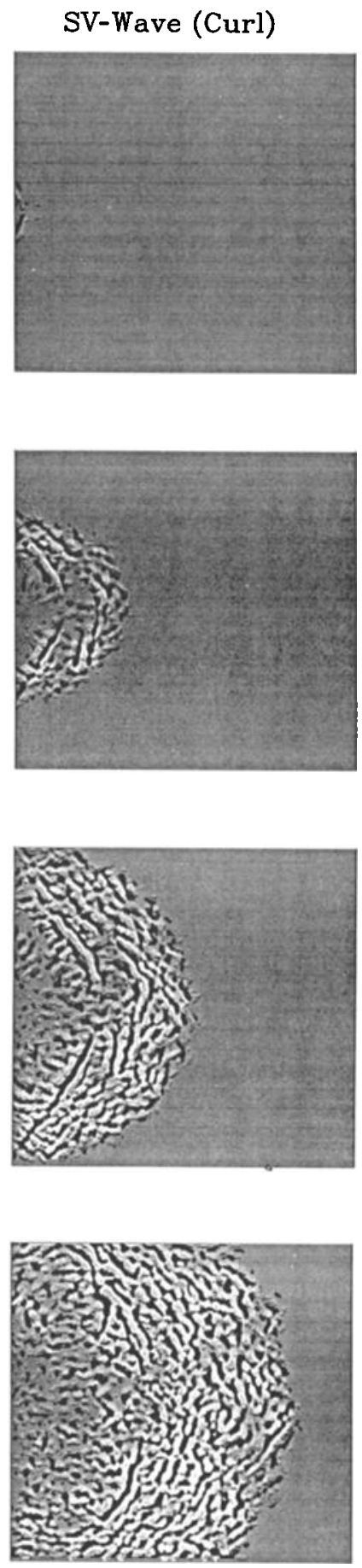

Fig. 3. Snapshots (time slices) of the divergence ( $P$ waves) and curl ( $S V$ waves) for a wave field propagating through the medium shown in Figure 2 with a standard deviation in velocity of $10 \%$. The $k a$ value at the dominant wavelength is about 1 . For each time step the divergence and curl are displayed with the same shading scale.

where $\lambda$ and $\mu$ are the Lamé constants and $\rho$ is the density. The solutions for these equations contain all converted phases, multiple reflections, and diffractions. These equations are solved numerically by replacing the partial derivatives by their finite difference approximations for a discrete time step $\Delta t$ and grid spacing $h$. Details of the method are given by Kelly et al. [1976]. The finite difference algorithms used in this paper utilized fourth-order approximations to the spatial derivatives, which are superior in accuracy to the second-order schemes more commonly used in finite difference modeling of seismic waves. The inaccuracy of the finite difference algorithms at high frequencies produces frequency dependent errors in phase and group velocity known as grid dispersion [Alford et al., 1974]. These errors are a function of the wavelength to grid spacing ratio and can produce effects in the synthetic seismograms similar to those of attenuation. Appendix A discusses various accuracy tests that we have performed to ensure that these errors are not contaminating our results.

The random media are characterized by their correlation function (or, equivalently, their fluctuation spectrum), prob- 
CONSTANT VEL
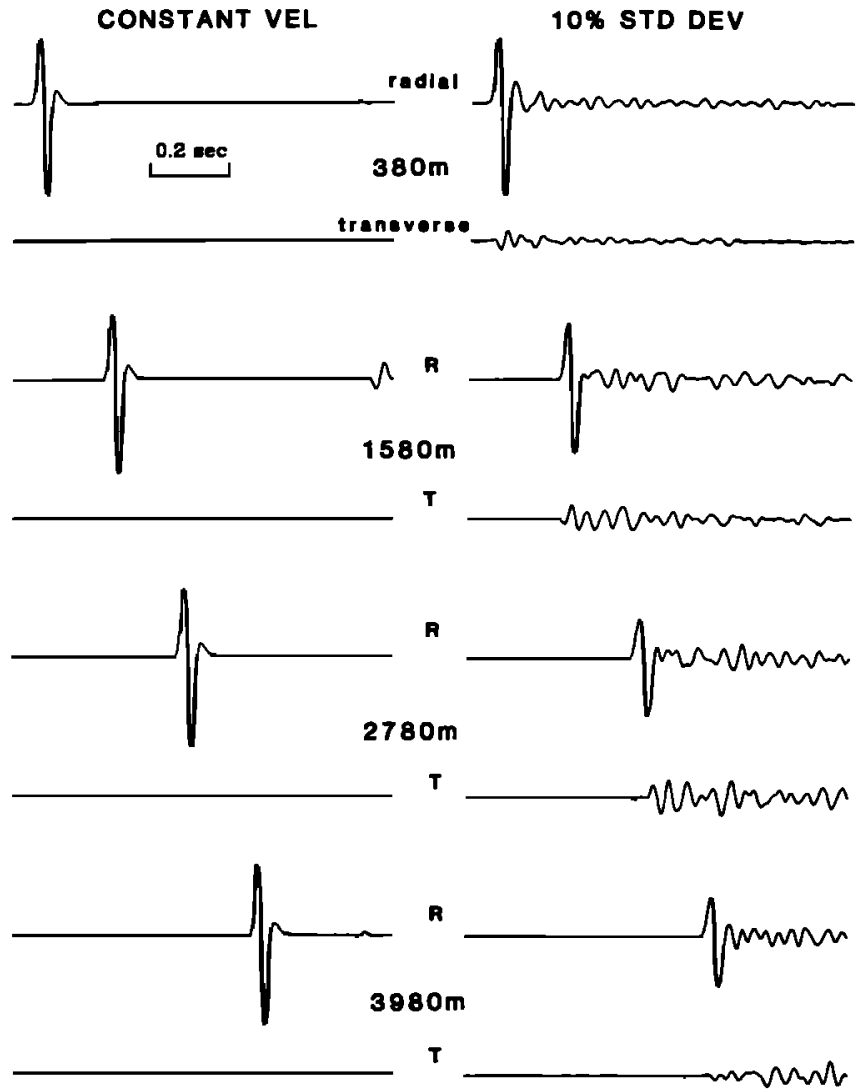

Fig. 4. (Left) Synthetic selsmograms for a $P$ wave propagating through a constant velocity medium (see Figure 2 for geometry). Radial and transverse components of velocity are shown for receivers from 380 to $3980 \mathrm{~m}$ from the source. Amplitudes of all synthetics shown in this paper are corrected for geometrical spreading. (Right) Synthetic seismograms for a $P$ wave traveling through an exponential random medium ( $\sigma_{c}=10 \%, a=40 \mathrm{~m}, k a=1.16$ at $\left.30 \mathrm{~Hz}\right)$.

ability distribution, and standard deviation (or spectral amplitude). Two correlation functions often used in scattering theory [Chernov, 1960] are the Gaussian function, where $N(r)=e^{-r^{2} / a^{2}}$ and the exponential function $N(r)=e^{-r / a}$. Here $r$ stands for the offset (or spatial lag) and $a$ is the correlation distance. The random medium is assumed to be isotropic. A third type of correlation function, the Von Karman function [Tatarski, 1961] commonly cited in turbulence theory, is defined as

$$
N(r)=\frac{1}{2^{m-1} \Gamma(m)}\left(\frac{r}{a}\right)^{m} K_{m}\left(\frac{r}{a}\right)
$$

where $K_{m}(x)$ is a modified Bessel function of order $m$ and $\Gamma(m)$ is the gamma function. In this paper, we consider a specific type of Von Karman function, where $m$ equals zero. For this case, the unnormalized correlation function is

$$
N(r)=K_{0}\left(\frac{r}{a}\right)
$$

The correlation functions used in this paper are listed in Table 1 along with their one- and two-dimensional Fourier transforms. The Fourier transform of the correlation function represents the power spectrum of the medium fluctuations. The one-dimensional Fourier transform $P(k)$ is given by

$$
P(k)=\int_{-\infty}^{\infty} N(r) e^{i k r} d r
$$

and the two-dimensional Fourier spectrum $P\left(k_{r}\right)$ is [Oberhettinger, 1972]

$$
P\left(k_{r}\right)=\int_{0}^{\infty} N(r) r J_{0}\left(k_{r} r\right) d r
$$

where $k_{r}$ is the radial wave number and equals $\left(k_{x}^{2}+{k_{z}}^{2}\right)^{1 / 2}$ and $J_{0}(x)$ is the zero-order Bessel function.

The fluctuation spectra for these media are flat up to a corner wave number inversely proportional to the correlation distance and then fall off at higher wave numbers $(k a>1)$. The key feature which distinguishes the three correlation functions is the falloff in spectral amplitude of the velocity fluctuations for wavelengths less than $2 \pi$ times the correlation distance (i.e., $k a>1$ ). The Gaussian medium has a strong, exponential decay of spectral amplitude, resulting in a smoothly varying medium (see Figure 1). For the exponential medium the two-dimensional spectrum decays as $k_{r}{ }^{-3}$ for $k a>1$, and this medium has more "roughness" at small length scales than the Gaussian medium (Figure 1).

The medium with the Von Karman function $(m=0)$ is characterized by heterogeneities that are self-similar for $k a>1$. This medium is "rougher" at small length scales than the exponential medium (Figure 1). In this paper we use the term "self-similar" to indicate that the standard deviation of the medium, calculated over equal logarithmic intervals of wave number, remains constant over a range of length scales. The variance $\left(\sigma^{2}\right)$ over a certain range of radial wave numbers $\left(k_{r_{1}}, k_{r_{2}}\right)$ is estimated by integrating the two-dimensional power spectrum of the medium

$$
\sigma^{2}=\int_{k_{r_{1}}}^{k_{r_{2}}} P\left(k_{r}\right) k_{r} d k_{r}
$$

For the Von Karman medium with $m=0$, the variance computed over an octave interval of wave number is (for $k_{r} a \gg 1$ )

$$
\sigma^{2}=\int_{k_{r}}^{2 k_{r}} \frac{k_{r} d k_{r}}{k_{r}^{2}}=\left.\ln \left(k_{r}\right)\right|_{k_{r}} ^{2 k_{r}}=\ln (2)
$$

Therefore the standard deviation is independent of wave number (or length scale) for $k a \gg 1$, and the medium is selfsimilar. Andrews [1980] used a comparable fluctuation spectrum to model random variations in seismic stress drop along a fault surface. This type of medium represents one of the fractal geometries described by Mandelbrot [1977]. The essential property of fractal media is that they display irregularity on all length scales. In a number of studies, Mandelbrot has analyzed the fractal geometry of a variety of natural processes. The spectral amplitudes of such fractal media are power functions of wave number [Mandelbrot and Wallis, 1969].

The following procedure was used to construct the random media on the two-dimensional grid for most of the finite difference simulations described in this paper. First, a random number generator assigned a velocity $v(x, z)$ sequentially to each point on the grid. The random velocity field was then Fourier transformed to wave number space, filtered to achieve the desired spectrum, and transformed back to the spatial domain to yield the velocity field for the simulations. All the random media discussed in this paper had a Gaussian probability distribution of seismic velocity, centered on the average velocity. The ratio of $P$ and $S$ wave velocity was kept constant, so that both velocities varied in the same manner across the grid. The density was constant at all points. The media were normalized by their standard deviation. In some cases, 
$a=10 \mathrm{~km}$

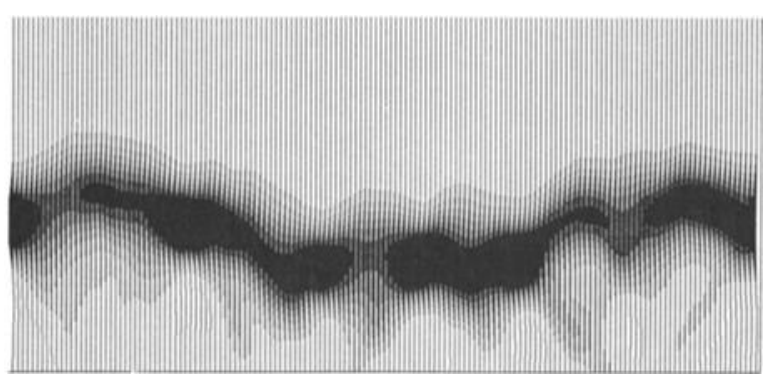

$0 \mathrm{sec}$

$2.8 \mathrm{sec}$

$0 \mathrm{~km}$

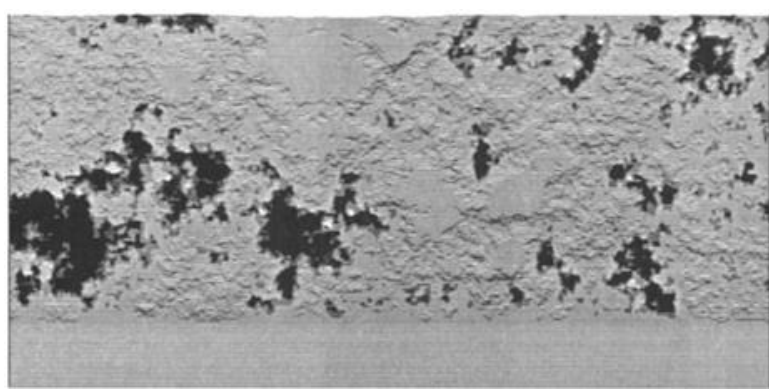

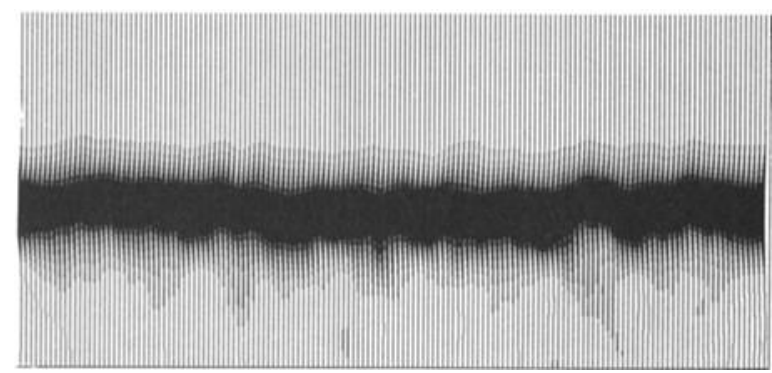

$a=1 \mathrm{~km}$

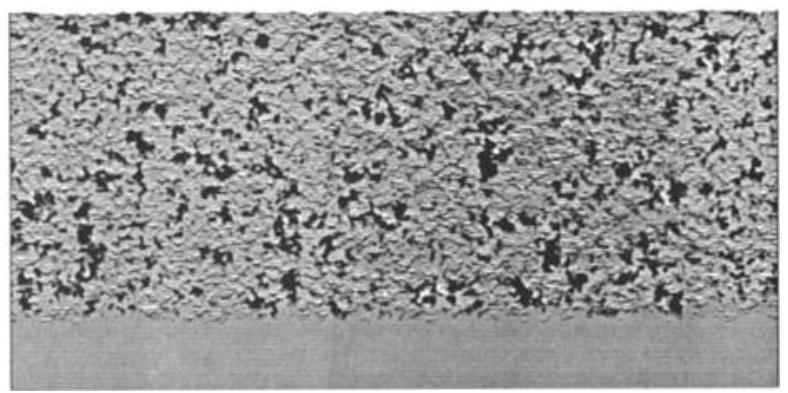

Fig. 5. (Top) Finite difference seismograms calculated for an array of receivers along the free surface for plane $P$ waves propagating vertically through (Bottom) random media with correlation distances of $10 \mathrm{~km}$ (left) and $1 \mathrm{~km}$ (right). Both media have exponential correlation functions and a standard deviation in velocity of $5 \%$. The plane wave is initiated in the homogeneous region at the base of the random medium. The seismograms are the vertical components, with positive displacements shaded, and are aligned at the positions of the receivers along the free surface.

the standard deviation of a discrete random medium differs significantly from that of a continuous medium with the same spectrum. The normalization of the random media is discussed in detail in Appendix B.

Figure 2 depicts a typical grid configuration used for the attenuation measurements described in a later section of this paper: a 210 by 200 point grid, $20 \mathrm{~m}$ grid spacing, average $P$ wave velocity of $6.5 \mathrm{~km} / \mathrm{s}$, average $S$ wave velocity of 3.65 $\mathrm{km} / \mathrm{s}$, density of $2.7 \mathrm{~g} / \mathrm{cm}^{3}$. The source is an explosion located near the left edge of the grid and consists of a line source extending perpendicular to the plane of the grid. The dominant frequency of the source wavelet is $20 \mathrm{~Hz}$. The medium has an exponential correlation function with $a=80 \mathrm{~m}$ so that $k a \approx 1$ at the dominant wavelength of the pulse. The standard deviation in velocity is $10 \%$. All edges of the grid have absorbing boundary conditions [Clayton and Engquist, 1977] to suppress artificial reflections.

In Figure 3 we show a series of time slices (or snapshots) of elastic waves propagating through this random medium. Many important features of wave propagation through random media are apparent. We display the divergence and curl of the seismic wave field in Figure 3 rather than the displacements, so that the $P$ wave and $S V$ wave energy can be discriminated. Since the source is an explosion, it generates only $\boldsymbol{P}$ waves (divergence). As the circular wave front propagates into the random medium, it becomes quite distorted. Significant differences in amplitude and travel time are produced for receivers at equal distances from the source. A large amount of $S V$ wave energy (curl) is observed behind the original $P$ wave front, a result of $P$ to $S V$ conversions during propagation through the random medium. The $P$ wave energy behind the original wave front is also caused by scattering. The scattered $S V$ wave energy in the time slices has shorter wavelengths than the scattered $P$ wave energy, a consequence of the slower velocity of the $S V$ waves. The semicircular $S V$ wave front generated at the left side of the grid (see time $\mathbf{0 . 0 6 4}$ s) is an artificial reflection of the $P$ wave traveling leftward from the source. This artifact travels well behind the initial $\boldsymbol{P}$ wave front and most of the scattered $S V$ wave energy.

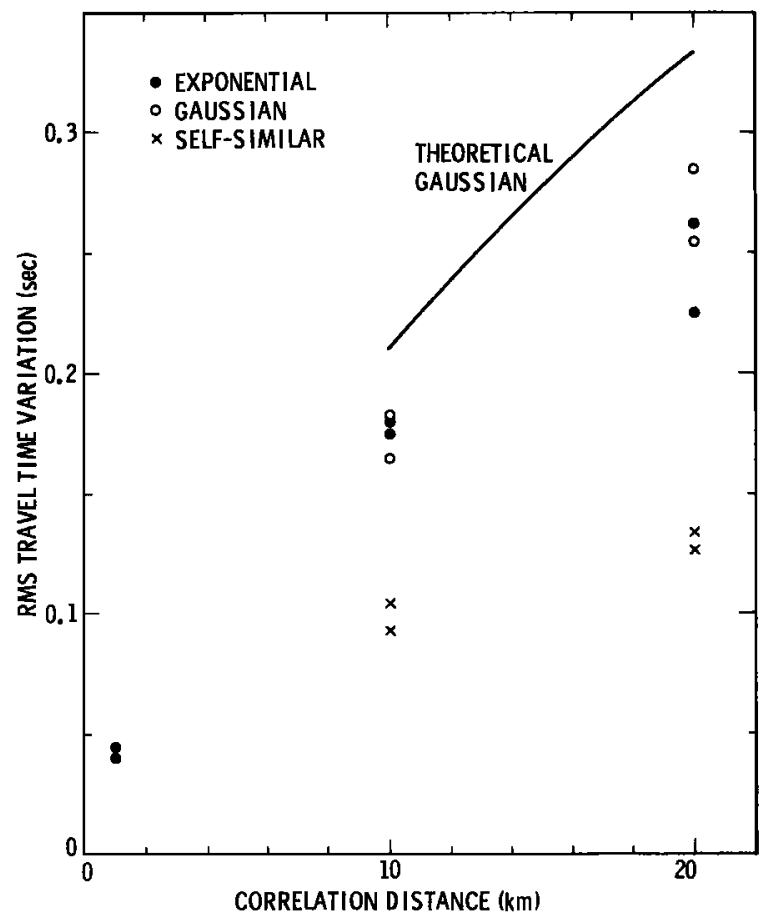

Fig. 6. Root mean square (rms) variation in travel time observed across the array in the simulations as a function of the correlation distance of the random media (5\% standard deviation in velocity). For each correlation distance, results are shown for media made from two different random number seeds. The solid curve represents the theoretical travel time variation for Gaussian media derived by Chernov [1960]. 


\section{a: $20 \mathrm{~km}$}

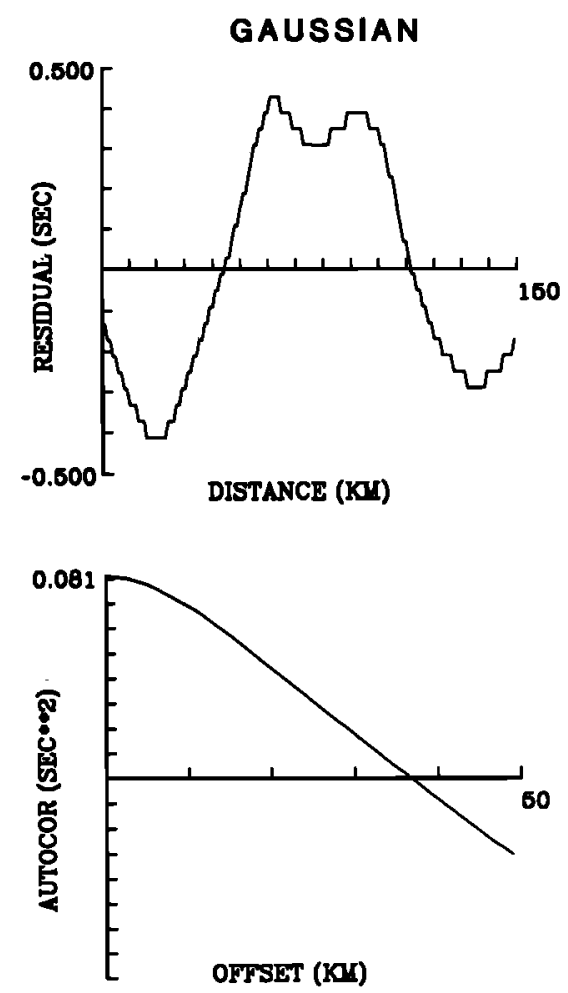

DISTANCE (KM)
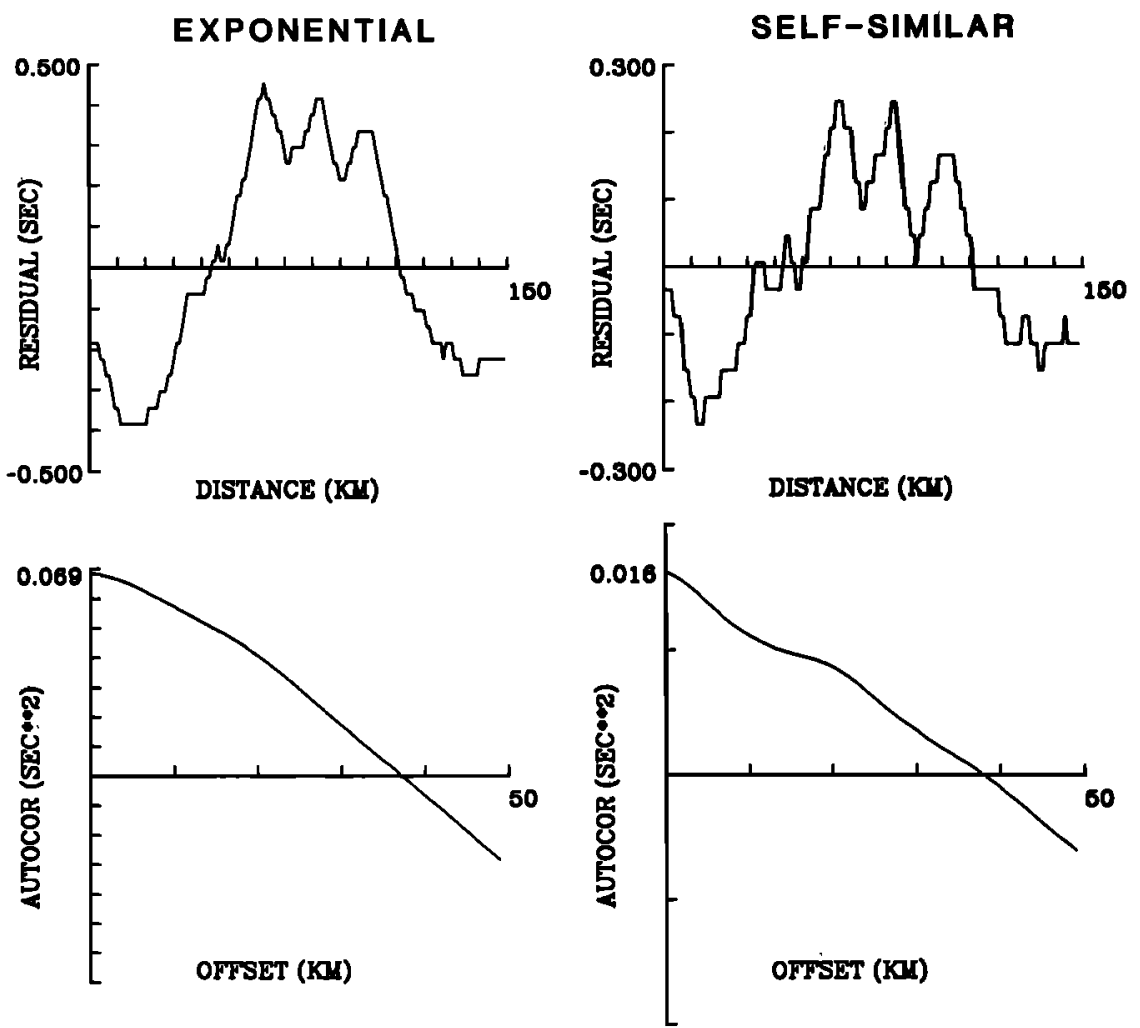

Fig. 7. (Top) Travel time residual plotted against distance along the free surface for Gaussian, exponential and self-similar random media with $a=20 \mathrm{~km}$ and $\sigma=5 \%$. The stairstep appearance of the anomalies is caused by the discrete time step used in the finite difference algorithm. (Bottom) Spatial autocorrelation functions of the travel time anomalies shown above, plotted on expanded distance scales.

Figure 4 displays the synthetic seismograms for receivers embedded within the finite difference grid, extending outward from the source. On the left side of Figure 4, synthetics for a $P$ wave pulse propagating through a constant velocity medium are shown. The amplitudes of all the synthetics displayed in this paper are corrected for geometrical spreading (multiplied by the square root of distance).

The synthetics on the right side of Figure 4 are for the random medium of Figure 2. Several features of the synthetics are noteworthy and are qualitatively similar to properties of actual short-period seismograms (see also Frankel and Clayton [1984]). There is significant seismic coda after the direct pulse. Sizeable displacements on the transverse components are observed in the $P$ wave synthetics for the random medium. In addition, it is clear that the amplitude of the seismic pulse decreases in amplitude as it propagates through the random medium, compared to the constant velocity case. This effect was noted for acoustic waves by Frankel and Clayton [1984] and is a result of apparent attenuation from scattering. Intrinsic attenuation was not included in these simulations, so that all attenuation observed is caused by scattering. This scattering attenuation will be measured in a later portion of this paper.

\section{Constraints on Models of Crustal Heterogeneity}

In this section of the paper we consider three observations from actual seismograms (travel time variation, coda amplitude, and waveform cross correlation) and their implications for the spectrum of velocity fluctuations in the crust. The finite difference simulations are applied to quantify these parameters as a function of the correlation distance and correlation function of the random media. The results from the simulations are then compared with some actual observations to constrain models of crustal heterogeneity.

\section{Travel Time Variations}

There is a considerable collection of observations documenting the variation of travel times across seismic arrays. We are concerned here with large-scale seismic arrays with station spacings greater than $10 \mathrm{~km}$ and apertures of about $100 \mathrm{~km}$ or more. Significant travel time variations are observed by these networks for both teleseismic $\boldsymbol{P}$ waves and $\boldsymbol{P}$ waves from local earthquakes. The rms travel time variation $\left(\sigma_{\Delta T}\right)$ observed at LASA, for example, is about $0.2 \mathrm{~s}$ for a given teleseism [see $A k i$, 1973]. Other arrays, such as NORSAR in Norway and SCARLET in southern California, display similar fluctuations in travel time (see Berteussen [1975] and Powell and Meltzer [1984] respectively), with peak to peak variations of several tenths of seconds. Many studies have inverted the arrival time variations to obtain three-dimensional models of velocity fluctuations for the crust and upper mantle (see, e.g., Aki et al. [1977] and Humphreys [1985]). Because of the station spacing and ray coverage, these inversions can resolve velocity variations on scale lengths of greater than $10 \mathrm{~km}$. Lateral heterogeneity in the crust is also apparent in travel time residuals from local earthquakes. Simultaneous inversion for local velocity structure and earthquake hypocenters reveals significant variations in crustal velocity over length scales of about $10 \mathrm{~km}$ 
a: $10 \mathrm{~km}$

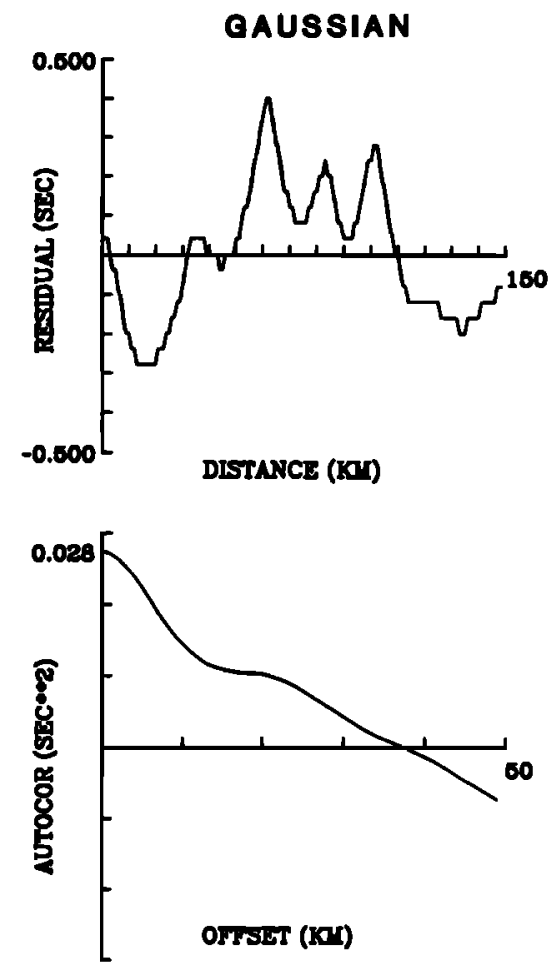

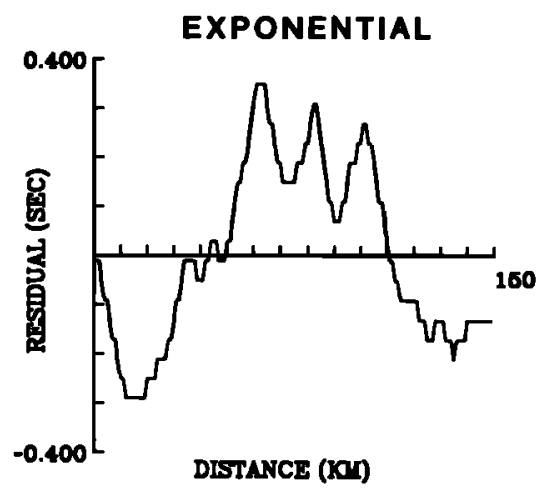

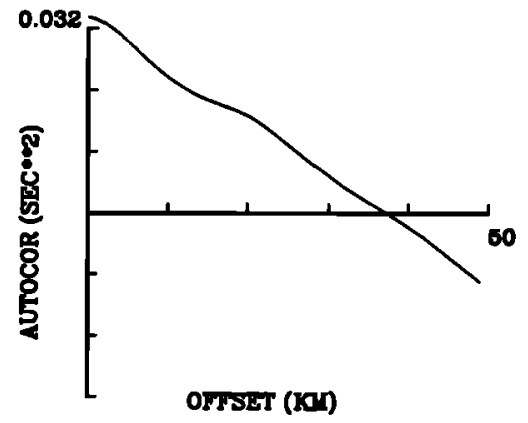

SELF-SIMILAR
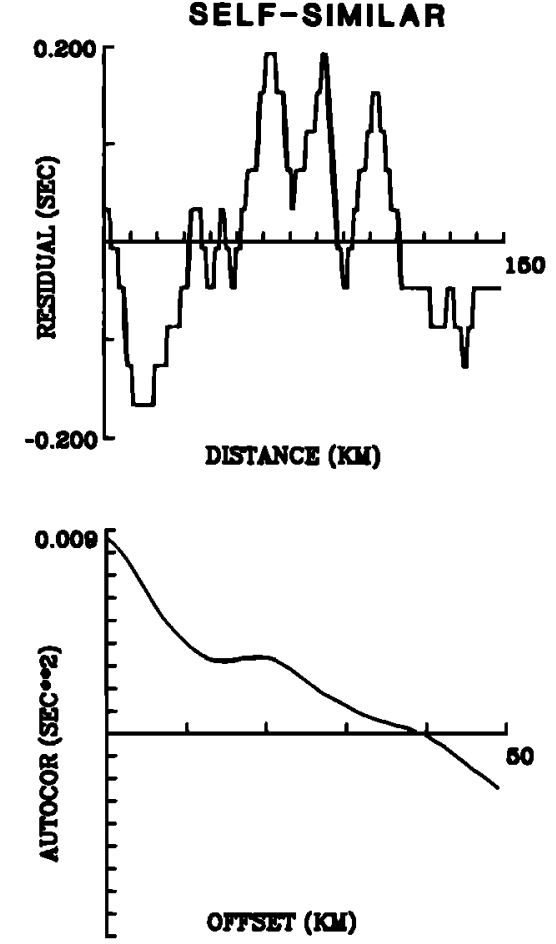

Fig. 8. Same as Figure 7 but with a correlation distance of $10 \mathrm{~km}$ for each medium.

(see, e.g., Thurber [1983]). Results from time term and tomographic studies from local earthquake data also document these larger-scale heterogeneities [e.g., Hearn and Clayton, $1986 a, b]$. Again, the reported peak to peak variations in travel time are several tenths of seconds. Any model of crustal heterogeneity must account for these variations of travel time.

Travel time variations for teleseismic waves propagating through random media were determined from the elastic finite difference synthetics. The geometry is shown in Figure 5. A horizontal plane $P$ wave is propagated vertically through the random medium, and seismograms are calculated for an array of 150 receivers spaced $1 \mathrm{~km}$ apart along the free surface. The grid spacing is now $500 \mathrm{~m}$, so that the vertical dimension of the random medium (110 grid points) corresponds to a thickness of $55 \mathrm{~km}$, somewhat larger than that of typical continental crust. The side and bottom edges of the grid have absorbing boundary conditions. To avoid edge effects, the actual side boundaries of the grid are located 50 grid points from either end of the array. The source wavelet is shaped as a Gaussian and has a dominant frequency of about $1 \mathrm{~Hz}$.

The simulations demonstrate that the amount of travel time fluctuation observed for a random medium is directly related to the correlation distance. Figure 5 shows the seismograms calculated for the array for exponential media with correlation distances of 10 and $1 \mathrm{~km}$, each with a $5 \%$ standard deviation in velocity. It is clear that the medium with the larger correlation distance produces much larger travel time variations ( $\mathrm{rms}$ travel time variation $\sigma_{\Delta T}$ of $0.179 \mathrm{~s}$ ) than the medium with the smaller scatterers $\left(\sigma_{\Delta T}=0.04 \mathrm{~s}\right)$. This observation is intuitively obvious, but the finite difference simulations allow the quantification of these travel times for a variety of random media. Figure 5 also illustrates that the medium with larger scatterers produces greater variations in amplitudes across the array.

Figure 6 displays the travel time variations observed in the simulations as a function of correlation distance, for Gaussian, exponential, and self-similar media. Travel times were determined from the time on the synthetic seismograms where the $P$ wave amplitude first exceeds 0.01 of the peak amplitude. The travel times were measured to an accuracy of $0.04 \mathrm{~s}$, which corresponds to the time step used in the finite difference scheme. The measurements were made for media constructed with two random number seeds. Figures $7-9$ show the travel time variations across the array for various media and will be discussed in detail below.

The results from the simulations demonstrate that a correlation distance greater than about $10 \mathrm{~km}$ is required to explain the rms travel time variations greater than $0.1 \mathrm{~s}$ that are observed in actual data (Figure 6). For all the media the rms travel time variations clearly increase with correlation dis-

TABLE 2. Measured Widths $l_{1 / 2}$ of Autocorrelation Functions of Travel Time Anomalies

\begin{tabular}{lccc}
\hline & & \multicolumn{2}{c}{$l_{1 / 2}, \mathrm{~km}$} \\
\cline { 3 - 4 } \multicolumn{1}{c}{ Medium } & $a, \mathbf{k m}$ & Medium A & Medium B \\
\hline Gaussian & 10 & 12 & 9 \\
Gaussian & 20 & 21.5 & 15 \\
Exponential & 1 & 3 & 3 \\
Exponential & 10 & 19 & 11 \\
Exponential & 20 & 23 & 14 \\
Self-similar & 10 & 9 & 8 \\
Self-similar & 20 & 21 & 11 \\
\hline
\end{tabular}


tance (Figure 6). While travel time variations for the Gaussian and exponential media with the same correlation distance $a$ are comparable, the self-similar medium with the same $a$ exhibits about half the fluctuation.

Figure 6 also shows the theoretical travel time variations predicted for Gaussian random media by the scalar theory of Chernov [1960]. Using the result for the variance of phase fluctuations $\sigma_{\Delta \phi}$ from Chernov [1960] and substituting

$$
\sigma_{\Delta T}=\frac{1}{k v_{0}} \sigma_{\Delta \phi}
$$

we find the variance of the travel time is

$$
{\sigma_{\Delta T}^{2}}^{2}=\frac{(\pi)^{1 / 2}}{2} \sigma^{2} \frac{a L}{v_{0}^{2}}\left(1+\frac{1}{D} \tan ^{-1} D\right)
$$

where $L$ is the thickness of the heterogeneous zone, $\sigma$ is the standard deviation in velocity, $v_{0}$ is the average velocity, $D=$ $4 L / k a^{2}$, and $k$ is the wave number (here $1 \mathrm{~km}^{-1}$ ). The theoretical travel time variations are similar to the observations from the synthetics for the Gaussian media.

The travel time fluctuation is observed to be dependent on the thickness of the heterogeneous zone $L$, for media with relatively large correlation distances $(\geq 10 \mathrm{~km})$. We have performed two finite difference runs for media with 220 grid points in the $z$ direction, corresponding to a thickness of 110 $\mathrm{km}$, twice that of the previous examples. For a self-similar medium with $a=20 \mathrm{~km}$, the rms travel time variation increased significantly from 0.128 to $0.148 \mathrm{~s}$ when the thickness was doubled. However, the observed increase of travel time with $L$ is less than the factor of 1.36 predicted by equation (9) for Gaussian media. For the case of an exponential medium with $a=1 \mathrm{~km}$, the travel time variation showed no dependence on the thickness of the medium, increasing by only $0.005 \mathrm{~s}$ as the thickness was doubled.

Thus the rms travel time anomalies observed at LASA and NORSAR $\left(\sigma_{\Delta T} \approx 0.2 \mathrm{~s}\right)$ can be produced by a self-similar medium with $a \geq 20 \mathrm{~km}$, a standard deviation of about $5 \%$, and a thickness somewhat greater than $100 \mathrm{~km}$. Of course, certain trade-offs occur in the estimation of these parameters, since increases in $a, L$, and $\sigma$ all produce greater travel time variations.

The spatial correlation of the travel time variations across large-scale arrays provides another key constraint on the correlation distance of the crust and upper mantle. The variations in travel time across the array used in the simulations are shown in Figures 7 and 8 for media with correlation distances of 20 and $10 \mathrm{~km}$, respectively $(L=55 \mathrm{~km}, \sigma=5 \%$ ). Each medium was produced with the same random number seed. In Figure $7(a=20 \mathrm{~km})$ we see a similar pattern in the long wavelength $(\approx 100 \mathrm{~km})$ anomaly between the Gaussian, exponential, and self-similar media, with slow travel times in the center of the array and fast arrivals at the edges. However, the travel time fluctuations at small length scales ( $\leq 30 \mathrm{~km}$ wavelength) are larger (relative to the long-wavelength anomaly) for the self-similar and exponential media than the Gaussian one. For any particular type of random medium, these smallscale variations are larger when $a=10 \mathrm{~km}$ than for $a=20 \mathrm{~km}$ (compare Figures 7 and 8).

The width of the spatial autocorrelation function of the travel time anomalies is observed in the simulations to be dependent on the correlation distance of the velocity vari-
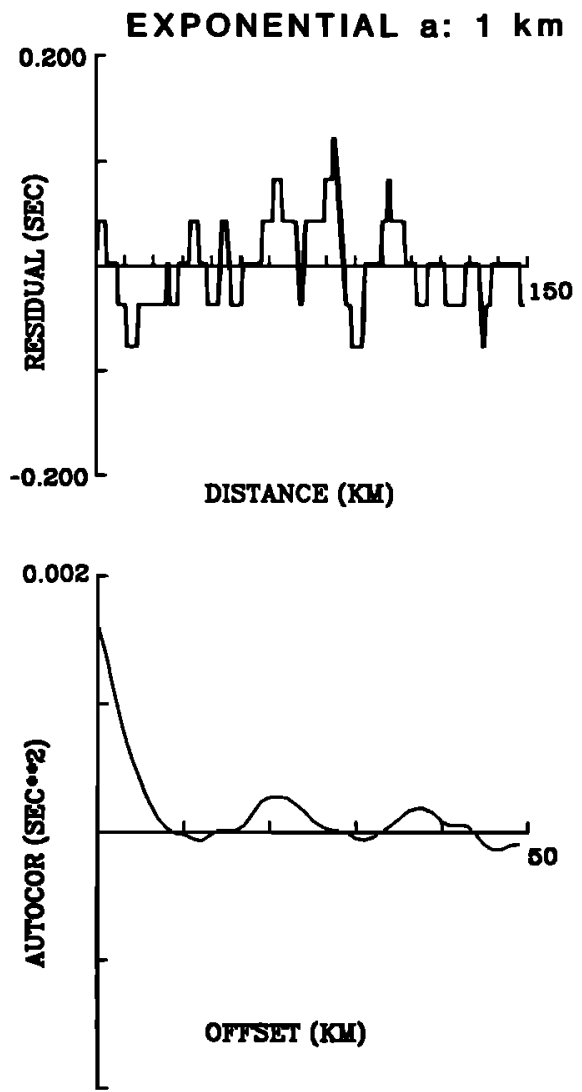

Fig. 9. Travel time residuals and autocorrelation function for an exponential medium with $a=1 \mathrm{~km}$ and $\sigma=5 \%$. The jagged appearance of the residuals is again the result of the discrete time step of the simulations.

ations of the medium. These autocorrelation functions are also depicted in Figures 7 and 8 . We measured the spatial $\operatorname{lag} l_{1 / 2}$, where the height of the autocorrelation function reaches half its peak value and the results are listed in Table 2 for media produced with two random number seeds. The simulations indicate that $l_{1 / 2}$ increases with $a$ for each type of medium and is roughly comparable to $a$ in the cases studied. This observation is consistent with the theoretical results of Chernov [1960], who found that $l_{1 / 2}$ is about equal to $a$ for Gaussian media (for $D \leq 10$ ). In the simulations, no systematic difference was observed in the values of $l_{1 / 2}$ between self-similar, exponential, and Gaussian media with the same correlation distance $a$.

An exponential medium with $a=1 \mathrm{~km}$ cannot produce the spatial correlation of travel time anomalies commonly observed in large-scale arrays. The travel time variations derived from the synthetics for such a medium are shown in Figure 9, along with their autocorrelation function. Again we note the small travel time variations for such a medium. The autocorrelation function is quite narrow, with $l_{1 / 2}=3 \mathrm{~km}$. This width is significantly less than those of correlation functions observed at LASA, NORSAR, GBA (India), and SCARLET, which are consistently greater than $10 \mathrm{~km}$ (see $A k i$ [1973], Berteussen et al. [1975a, b], and Powell and Meltzer [1984], respectively). The finite difference results demonstrate that selfsimilar, exponential, and Gaussian random media with $a \geq 10$ $\mathrm{km}$ produce autocorrelation functions with widths of $8 \mathrm{~km}$ and larger (Figures 7 and 8), similar to the actual observations. 


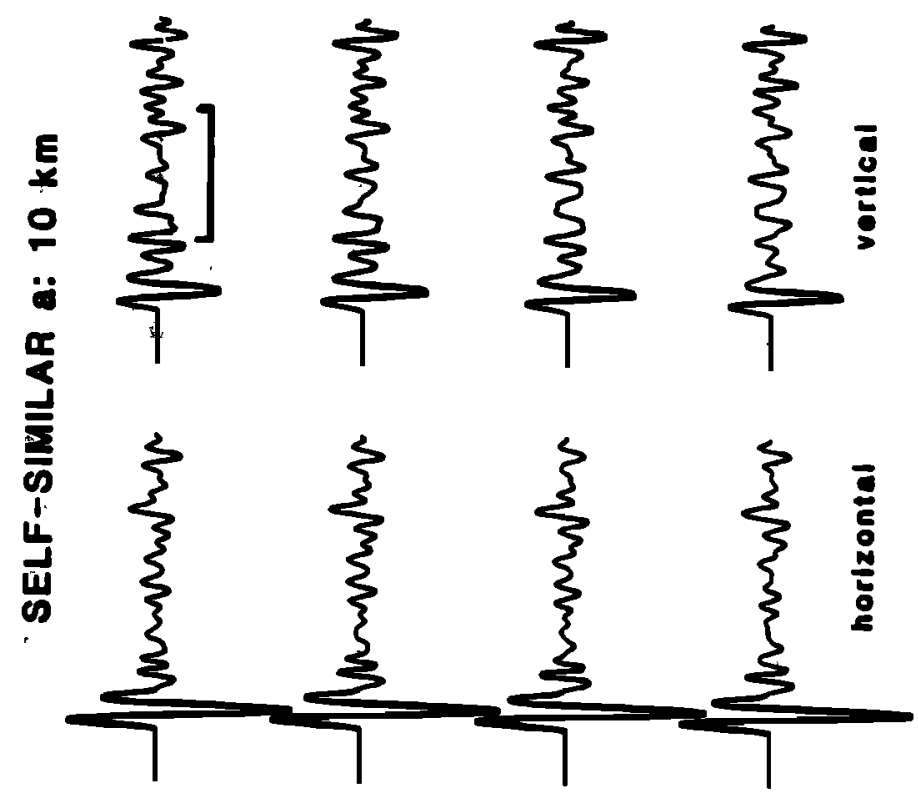

으료뫃

롭

ह

惫虽

预总焉

兘的卷

의 8

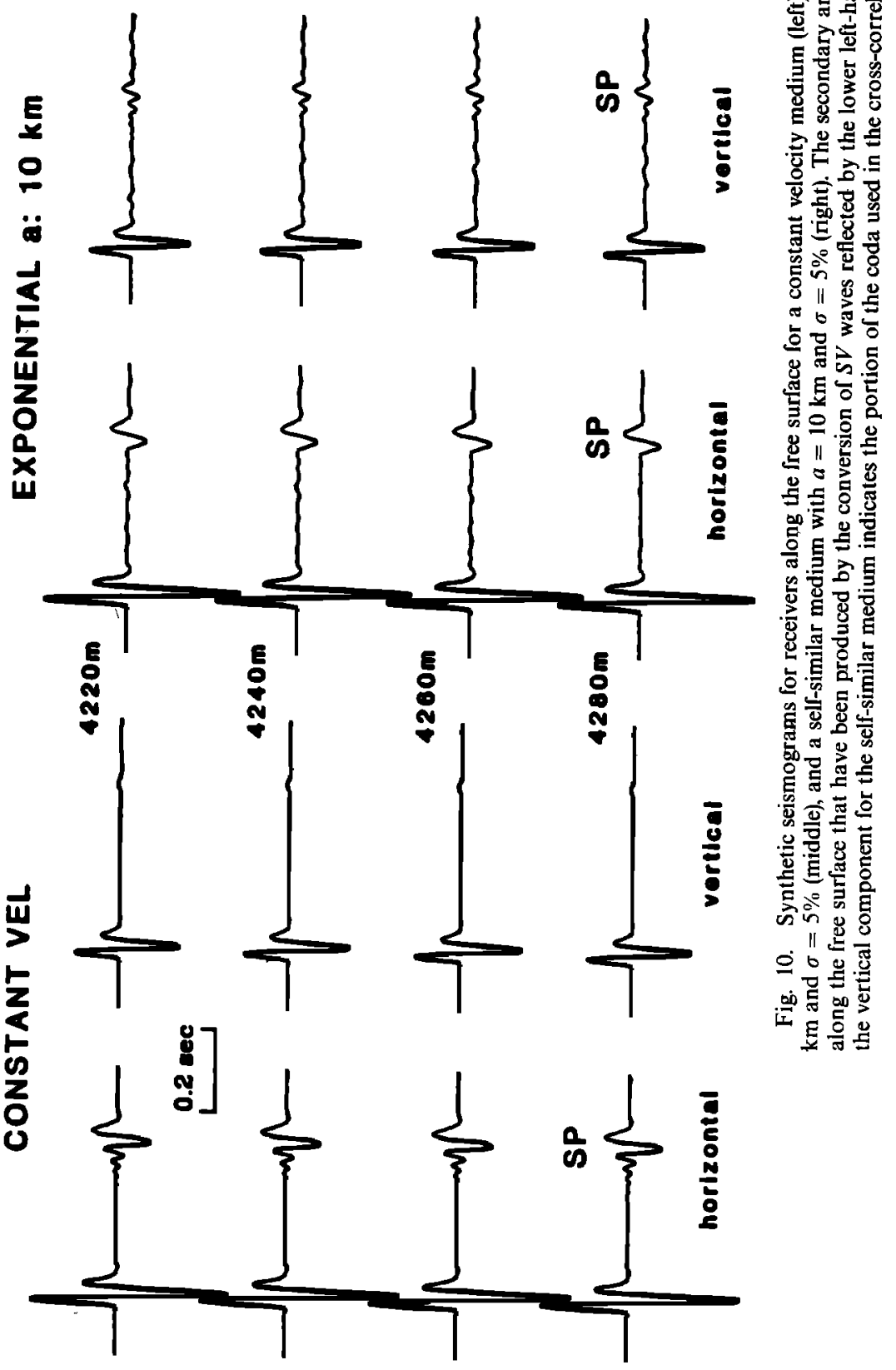




\section{Coda Amplitude at High Frequencies}

Another major constraint on the heterogeneity spectrum of the crust is the presence of seismic coda at high frequencies $(>10 \mathrm{~Hz}$ ) in the waveforms of microearthquakes. We quantify the coda amplitude and waveform variation for finite difference synthetics obtained with the configuration shown in the top panel of Figure 1. This configuration is intended to approximate a geometry common to local recordings of microearthquakes, with receivers at an epicentral distance about twice the source depth. For the simulations a group of receivers are placed along the free surface, starting at an epicentral distance of $3.2 \mathrm{~km}$ and spaced $20 \mathrm{~m}$ apart (one grid spacing). An explosion source is located at $2 \mathrm{~km}$ depth in the lower left-hand corner of the grid. The dominant frequency of the source is about $20 \mathrm{~Hz}$. The left, right, and bottom edges of the grid all have absorbing boundary conditions.

The finite difference synthetics document that the amplitude of the high-frequency coda is dependent on the falloff of the heterogeneity spectrum at high wave numbers. Figure 10 illustrates the synthetic seismograms for self-similar and exponential media with a correlation distance of $10 \mathrm{~km}$ and a standard deviation in velocity of $5 \%$. These random media, with correlation distances larger than the total grid size, were constructed by the procedure described in Appendix B. It is clear from Figure 10 that the self-similar medium with $a=10$ $\mathrm{km}$ produces significant coda at frequencies of $30 \mathrm{~Hz}$, whereas the exponential medium does not. The $P$ wave coda amplitude for the self-similar medium is roughly comparable to that observed in microearthquake waveforms recorded at similar hypocentral distances $(\approx 5-10 \mathrm{~km})$ in a variety of tectonic regimes. The reader is referred to seismograms displayed by Fletcher [1980, 1982] and Aki and Chouet [1975] for microearthquakes near Oroville, California; Monticello, South Carolina; and NORSAR in Norway, respectively. The relative amplitude of the direct arrival and the coda will be affected by the radiation pattern of the earthquake source, which is not included in these simulations (isotropic explosion source). However, this effect does not bias our comparison between the synthetics and microearthquake waveforms, since we are citing a general observation based on a number of microearthquake waveforms. Any variation in the relative amplitude of the $P$ wave and the coda caused by the earthquake radiation pattern would be averaged out over the many observations of microearthquake waveforms.

We examined the amplitude of the synthetic coda as a function of correlation distance for self-similar and exponential media. Typical synthetic seismograms for self-similar and ex-

\section{SELF-SIMILAR dist: $3740 \mathrm{~m}$ EXPONENTIAL}

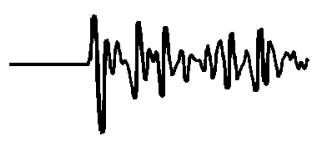

a: $200 \mathrm{~m}$
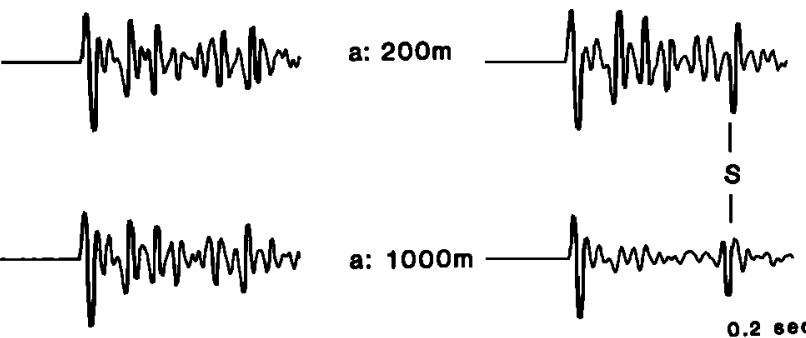

$0.2 \mathrm{Bec}$

Fig. 11. Synthetic seismograms (vertical components) for a receiver at the free surface for self-similar and exponential media with correlation distances of $200 \mathrm{~m}$ and $1000 \mathrm{~m}(\sigma=10 \%)$. "S" indicates the $S V$ wave reflected from the lower left-hand corner of the grid.

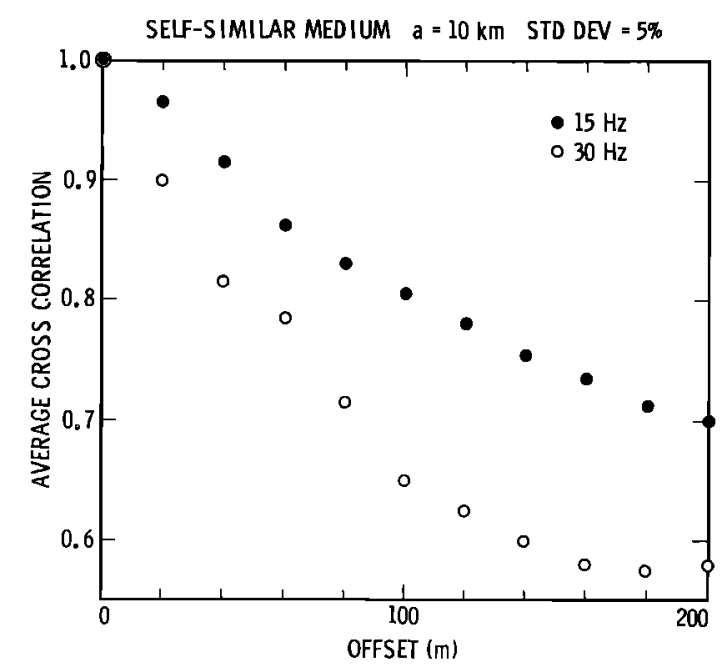

Fig. 12. Average of the cross correlation of the filtered synthetic coda (see Figure 10) as a function of receiver separation or offset (see text). Results are shown for synthetics band-pass filtered at 12.5-17.5 $\mathrm{Hz}$ and 25-35 $\mathrm{Hz}$. The medium was self-similar with $a=10 \mathrm{~km}$ and $\sigma=5 \%$.

ponential media $(\sigma=10 \%)$ with $a=200 \mathrm{~m}$ and $1000 \mathrm{~m}$ are shown in Figure 11 (geometry as in Figure 1). The coda amplitude decreases strongly for the exponential medium as the correlation distance increases. In contrast, the coda amplitude remains about constant for the self-similar media, despite the factor of 5 increase in $a$. The rms amplitude of the filtered coda $(25-35 \mathrm{~Hz})$ was measured and compared for the vertical component at 20 receivers along the free surface. The ratio of the coda amplitude between the exponential media with $a=1000 \mathrm{~m}$ and $200 \mathrm{~m}$ was $0.46 \pm 0.06$.

This behavior of the coda amplitude with respect to correlation distance is consistent with the weak-scattering theory of Chernov [1960]. We adapt this theory to the two-dimensional geometry of the simulations in Appendix C. For two random media with correlation distances $a_{1}$ and $a_{2}$ (equal $\sigma$ ), equation (C7) allows the ratio of scattered energy $\left(\Phi_{1} / \Phi_{2}\right)^{2}$ at wave number $k$ and angle $\theta$ to be expressed as the ratio of the two-dimensional power spectra $\left(P\left(k_{r}\right)\right)$ of the media, such that

$$
\left(\frac{\Phi_{1}}{\Phi_{2}}\right)^{2}=\frac{P_{1}[2 k \sin (\theta / 2)]}{P_{2}[2 k \sin (\theta / 2)]}
$$

Substituting the power spectrum for exponential media (Table 1) into (15), the ratio of the scattered amplitude becomes

$$
\frac{\Phi_{1}}{\Phi_{2}}=\left[\left(\frac{a_{1}}{a_{2}}\right)^{2} \frac{\left(1+k_{m}{ }^{2} a_{2}{ }^{2}\right)^{3 / 2}}{\left(1+k_{m}{ }^{2} a_{1}{ }^{2}\right)^{3 / 2}}\right]^{1 / 2}=\left(\frac{a_{2}}{a_{1}}\right)^{1 / 2}
$$

where $k_{m}=2 k \sin (\theta / 2)$ and $k_{m} a \gg 1$. Thus the coda amplitude for exponential media will be inversely proportional to the square root of the correlation distance, as is roughly found in the amplitudes of the synthetic coda noted above for $a_{1}=$ $1000 \mathrm{~m}$ and $a_{2}=200 \mathrm{~m}$.

In two self-similar media with correlation distances of $a_{1}$ and $a_{2}$, the ratio of coda amplitudes is found from equations (C10) and (10):

$$
\frac{\Phi_{1}}{\Phi_{2}}=\left[\left(\frac{a_{1}}{a_{2}}\right)^{2} \frac{\left(1+k_{m}{ }^{2} a_{2}^{2}\right)}{\left(1+k_{m}{ }^{2} a_{1}{ }^{2}\right)}\right]^{1 / 2}
$$

For $k_{m} a \gg 1$, this ratio approaches unity and the coda amplitude for self-similar media remains about constant with re- 


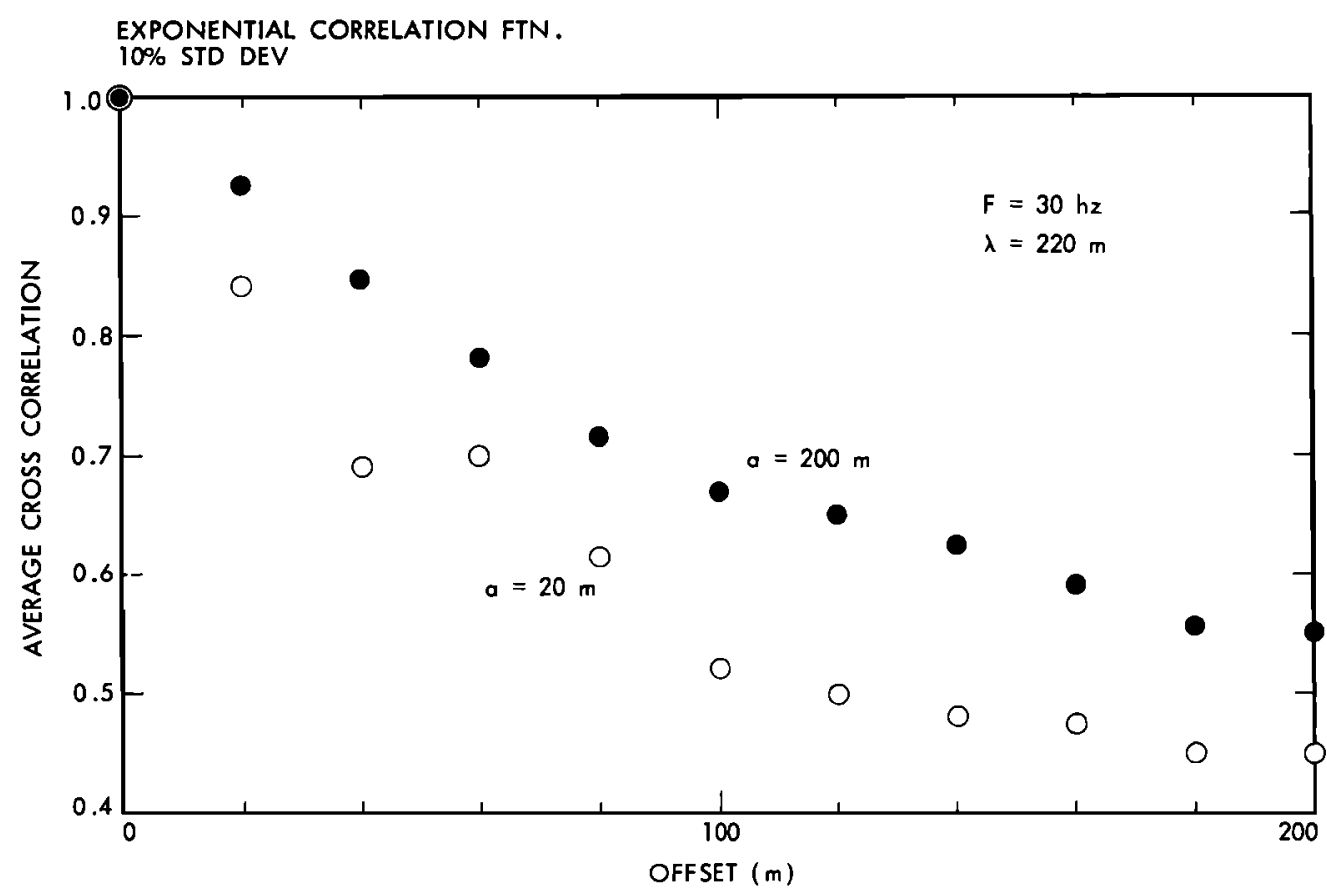

Fig. 13. Average of the cross correlation of the filtered $(25-35 \mathrm{~Hz})$ synthetic coda as a function of receiver separation for two exponential random media $(\sigma=10 \%)$ with differing correlation distances $(20 \mathrm{~m}$ and $200 \mathrm{~m})$.

spect to the correlation distance, as is observed in the simulations (Figure 11). Here we have also assumed that $k_{N y q} a \gg 1$, so that the normalization factor $C$ in equation (B9) is insensitive to the correlation distance.

The observations of the synthetic coda amplitudes lead to an important conclusion concerning the correlation function appropriate for the crust. Media with Gaussian and exponential correlation functions and correlation distances of 10 $\mathrm{km}$ or more (5-10\% standard deviation) would produce negligible amounts of coda at frequencies of $30 \mathrm{~Hz}$. However, these large correlation distances are required to explain the standard deviation and spatial correlation of travel time variations across large-scale seismic arrays. Gaussian and exponential random media with reasonable standard deviations in velocity $(\leq 10 \%)$ do not appear to satisfy both the travel time and coda observations.

A self-similar random medium can account for these two sets of observations. Such a medium would have a correlation distance $a$ of at least $10 \mathrm{~km}$ and a standard deviation in velocity of about $5 \%$.

\section{Waveform Variation Across Receiver Arrays}

Another useful observation that provides constraints on crustal heterogeneity is the variation of waveforms across seismic arrays. We quantify this waveform variation by determining the cross-correlation functions from the finite difference synthetics. As shown in Figure 10, a portion of the synthetic coda was windowed from the vertical components for 60 receivers along the free surface (see top panel of Figure 1 for geometry). The coda from each receiver was band-pass filtered and cross-correlated with that from every other receiver. The maximum of the cross-correlation function was determined for each pair of receivers and then averaged as a function of receiver separation or offset.

Figure 12 illustrates the results of this procedure for a selfsimilar medium with $a=10 \mathrm{~km}$ and a standard deviation of
$5 \%$. Each data point represents an average of from 50 to 59 determinations of cross-correlation maxima. The cross correlation decreases as the receiver separation increases. The rate of falloff of the cross correlation is greater at higher frequency $(30 \mathrm{~Hz})$ than at the lower frequency $(15 \mathrm{~Hz})$. These general features of the waveform cross correlation are commonly reported in actual data from small-scale arrays of seismometers with station separations of about $100 \mathrm{~m}$ [Smith et al., 1982; McLaughlin et al., 1983]. It is noteworthy that a self-similar medium with $a=10 \mathrm{~km}$ and $5 \%$ standard deviation produces significant variations in waveforms at frequencies of $15-30 \mathrm{~Hz}$ for receivers separated by more than $200 \mathrm{~m}$.

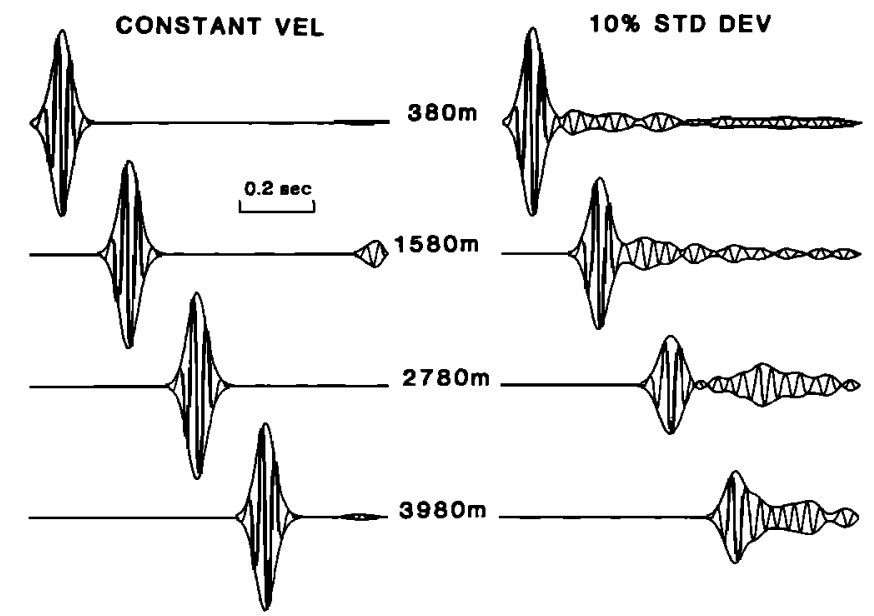

Fig. 14. Band-pass-filtered $(25-35 \mathrm{~Hz})$ and enveloped synthetic seismograms (radial components) for $P$ waves propagating through a constant velocity medium (left) and a random medium (right). Unfiltered seismograms are shown in Figure 4. Seismogram amplitudes are corrected for geometrical spreading. The scattering attenuation is determined from the decay of the peak envelope amplitude as a function of distance. 

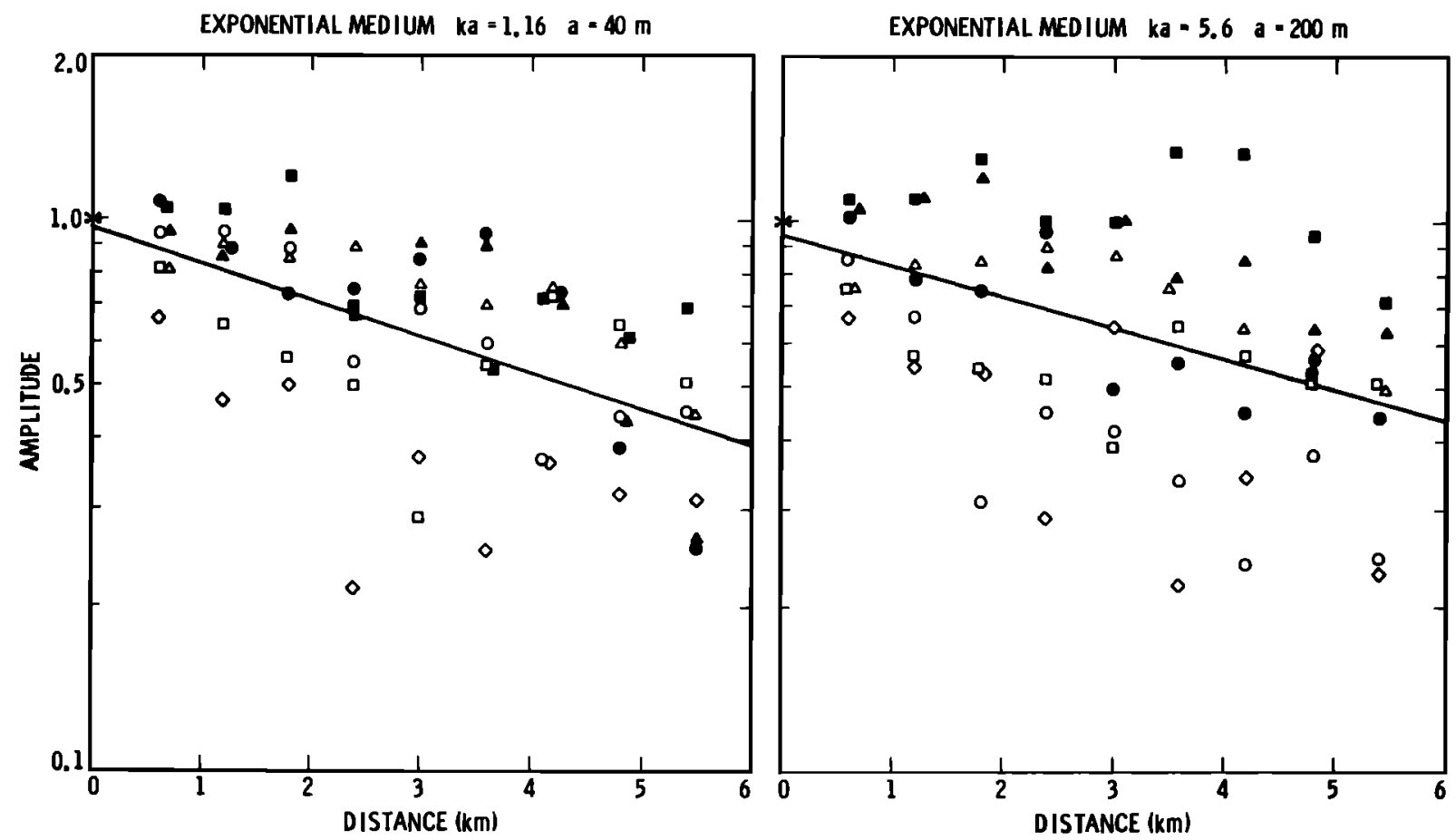

Fig. 15. Logarithm of the peak amplitude $(25-35 \mathrm{~Hz})$ plotted against distance for exponential media with correlation distances of $40 \mathrm{~m}$ (left) and $200 \mathrm{~m}$ (right), $\sigma_{c}=10 \%$. Amplitudes are normalized to the peak amplitude observed at the receiver nearest the source $(r=380 \mathrm{~m})$. Each type of symbol corresponds to measurements from a linear array of receivers along a particular azimuth from the source (see Figure 2). The lines denote the least squares fits to the amplitude decay used to estimate the scattering $Q$.

The synthetic seismograms also demonstrate that the rate of decrease of the cross correlation with offset is dependent on the characteristics of the random medium. In Figure 13, the cross-correlation results for exponential media ( $\sigma=10 \%$ ) with correlation distances of 20 and $200 \mathrm{~m}$ are depicted. The cross correlation decays much more rapidly with receiver separation for the medium with the shorter correlation distance.

These findings have implications to the reciprocal problem of analyzing the waveforms from closely spaced earthquakes at a single receiver. Many investigators have noted clusters of microearthquakes in many regions with virtually identical waveforms up to frequencies as high as $30 \mathrm{~Hz}$ [Geller and Mueller, 1980; Frankel, 1982a; Spieth, 1981; Pechmann and Kanamori, 1982]. Geller and Mueller [1980] proposed that the similarity of waveforms requires the spatial separation of these events to be less than one quarter of the seismic wavelength. The results in Figure 13 document that estimates of the maximum separation of similar earthquakes are dependent on the properties of the medium and are not simply a fixed fraction of the wavelength.

\section{Apparent Attenuation in Random Media}

In this section we use measurements from the finite difference synthetics to quantify the apparent attenuation from scattering for various types of random media. The apparent $Q$ is determined from the decay in peak amplitude of the bandpass-filtered synthetics with propagation distance. This yields an estimate of the apparent attenuation $\left(Q^{-1}\right)$ of the initial part of the waveform in a narrow frequency range. For each finite difference experiment we extract synthetic seismograms from several arrays of receivers extending radially outward from the source (see Figure 2). The synthetics are corrected for geometrical spreading, band-pass filtered $(25-35 \mathrm{~Hz})$ with phaseless Butterworth filters, and enveloped. An example of one profile of filtered records is shown in Figure 14.

The scattering $Q$ is determined from the least squares fit from graphs such as those in Figure 15. The log peak amplitudes of the filtered displacements are plotted as a function of distance for $P$ waves propagating through two random media. In both media there is an overall decrease of amplitude with increasing distance. However, considerable variation is found in the amplitudes measured at any given distance. This variation is larger for the medium with $k a=5.6$ (scatterers larger than the wavelength), where the amplitude variation reaches a factor of 6 at some distances. Certain paths through this random medium exhibit virtually no attenuation, whereas others have $Q$ values as low as about 60 . At several receivers in this medium, the amplitudes are greater than those in the constant velocity case. This indicates that multipathing is occurring. Obviously, fitting a linear decay to these amplitudes characterizes the scattering attenuation in only a gross sense for this medium. Finite difference runs with larger grid sizes are necessary to better separate the effects of multipathing and scattering attenuation for media with $k a \gg 1$, where relatively few scatterers are contained in the grid. The large variance in plots such as Figure 15 illustrates the difficulties of quantifying the attenuation in scattering media, difficulties frequently encountered in amplitude measurements of actual short-period seismograms.

We investigate the variation of scattering $Q$ as a function of the normalized frequency $k a$, by examining attenuation for $30-\mathrm{Hz} P$ waves propagating through random media with various correlation distances. Since $k a$ is proportional to frequency times the correlation distance, such a procedure essentially determines the frequency dependence of apparent attenuation in a particular random medium. For $30-\mathrm{Hz} P$ 


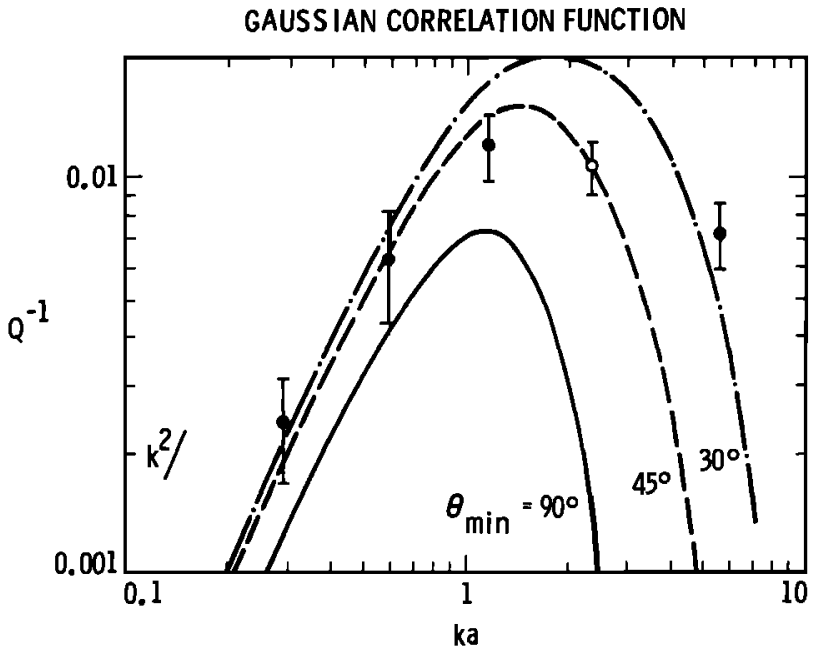

Fig. 16. Attenuation $\left(Q^{-1}\right)$ measured from the synthetics plotted as a function of $k a$ for Gaussian random media with standard deviation $\sigma_{c}$ of $10 \%$. Error bars represent one standard deviation, as calculated from the regression analysis. Solid circles denote the average of results from media with two random number sequences. Determinations from one medium are depicted with open symbols. The curves indicate the theoretical attenuation derived from Appendix C. $\theta_{\min }$ denotes the minimum scattering angle considered in the attenuation calculation.

waves, the waves propagate at least 17 cycles within the random medium (for the 210 by 200 point grid). It is necessary to observe the amplitude decay over this number of cycles to obtain accurate estimates of $Q$. An alternative method of estimating the frequency dependence of $Q$ would be to measure attenuation as a function of frequency for one medium. This is not practical because the attenuation of the low-frequency components cannot be reliably estimated due to the small number of wavelengths they travel through the grid. In many cases, $Q$ values were determined at a given $k a$ from synthetics produced in two random media, each characterized by the same power spectrum but different random number sequences (or seeds). The grid size for the runs with $k a \leq 1.16$ was 210 by 200 grid points. For runs with $k a=2.32$ and 5.6 (Gaussian and exponential media), the grid size used was increased to 300 by 300 points.

Figure 16 shows the attenuation $\left(Q^{-1}\right)$ values derived from the finite difference synthetics as a function of $k a$ for random media with Gaussian correlation functions. The apparent attenuation peaks at $k a$ between 1 and 2 . This essentially means that the scattering attenuation is greatest when the seismic wavelength is comparable to the size of the scatterers. This observation was also noted in the acoustic finite difference simulations reported by Frankel and Clayton [1984]. There is a rapid increase of attenuation with $k a$ for $k a<1$ and a gradual decline of attenuation at high frequencies $(k a>2)$.

It is useful to compare the results from the numerical simulations with predictions from the weak scattering theory described in Appendix C. Theoretical curves for scattering attenuation in a two-dimensional Gaussian random medium are depicted in Figure 16. These curves were derived from equation (C11), for three values of the minimum scattering angle $\theta_{\min }$. With the weak scattering theory, the appropriate value of the minimum scattering angle is unclear, since waves scattered in the forward direction reach the receiver at about the same time as the direct wave and should not be counted as lost energy [Sato, 1977; Wu, 1982; Malin and Phinney, 1985]. One of the motivations for using the finite difference synthetics to determine attenuation is to provide some constraint on the proper value for $\theta_{\text {min }}$.

The predicted attenuation is peaked at $k a$ between 1 and 2, in agreement with the results from the numerical simulations. The theoretical attenuation at $k a<1$ is proportional to $(k a)^{2}$, in correspondence with the results from the synthetics. We should note that the theoretical frequency dependence of attenuation for $k a \ll 1$ (the Rayleigh scattering limit) differs between two- and three-dimensional random media [see Menke, 1984]. Attenuation is proportional to $(k a)^{3}$ for $k a \ll 1$ in threedimensional media. The theory predicts that the amount of attenuation and the precise value of $k a$ at the peak attenuation are dependent on $\theta_{\text {min }}$. The high-frequency behavior of the attenuation $(k a \gg 1)$ is identical between the two- and three-dimensional theories.

The measured attenuation for Gaussian media cannot be matched by any single curve derived from the weak scattering theory for the range of $k a$ between 0.3 and 5.6. For $k a>1$ the observed decline of attenuation with increasing $k a$ is much more gradual than predicted by the theory. The significance of this difference is, at present, unclear. Because of multipathing, it is difficult to measure the attenuation for media with $k a>1$, so that the attenuation observations for these media may be unrepresentative. Alternatively, the discrepancy may be caused by the presence of multiple scattering and wave conversions neglected by the Chernov [1960] theory. Simulations involving larger grids will be needed to resolve this question.

Figure 17 shows the observed scattering attenuation for random media with exponential correlation functions, along with the theoretical attenuation curves. The observed attenuation increases as $k a$ increases from 0.3 to 1.2 . For $k a$ between 1.2 and 5.6 the attenuation remains about constant, in contrast to the Gaussian media. The observed attenuation for the exponential media roughly follows the theoretical curve for $\theta_{\min }=30^{\circ}$.

The observed and theoretical attenuation values for the selfsimilar random media are depicted in Figure 18. For these media we have measured the attenuation only for $k a \geq 1$. The

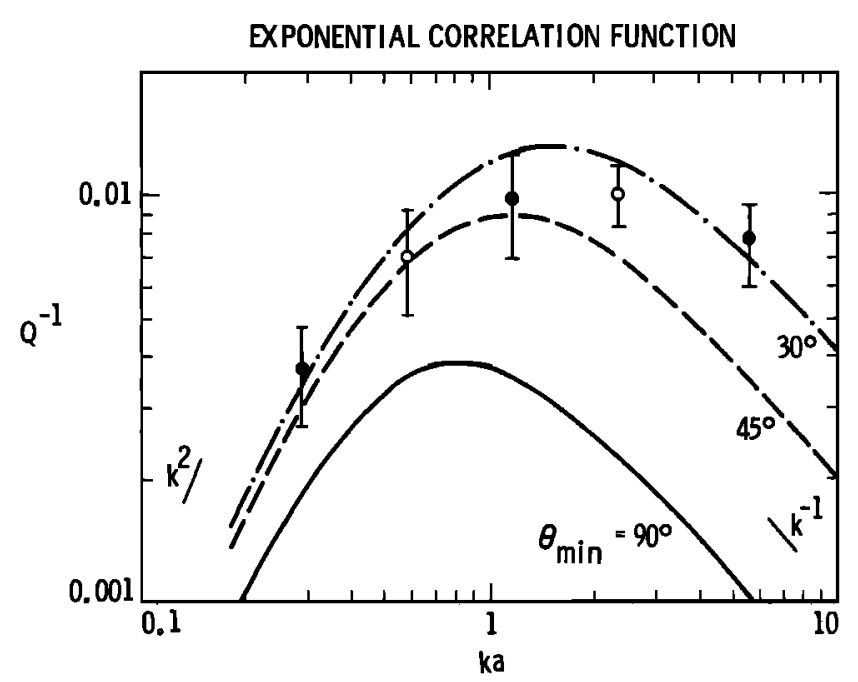

Fig. 17. Measured attenuation and theoretical curves for exponential random media, $\sigma_{c}$ of $10 \%$. 
attenuation observed in the numerical simulations is essentially constant with frequency. The theoretical attenuation curves for a $\theta_{\operatorname{man}}$ of $30^{\circ}$ match the numerical results for these media.

Although the finite difference simulations allow us to determine the overall frequency dependence of the scattering attenuation, more work is needed to quantify the absolute values of attenuation. Simulations involving several random media with different random number sequences (but identical power spectra) are required to achieve better constraints on the range of attenuation at any given $\mathrm{ka}$. We have found that varying the random number seed can produce differences in the absolute $Q$ determinations up to a factor of 1.5 .

The key parameter which distinguishes the theoretical attenuation behavior of the exponential and self-similar media is the Irequency dependence of $Q$ at high frequencies $(k a \gg 1)$. The independence of $Q$ with frequency for the self-similar medium is a direct consequence of the velocity fluctuations having equal amplitudes (or variance) over a broad range of length scales (see equation (8)). The apparent attenuation of the exponential medium decreases with frequency because the fluctuation amplitude of this medium decreases as the length scale gets smaller. The theoretical relationship between the frequency dependence of $Q$ and the fluctuation spectrum of the medium has been noted for Gaussian and exponential media by Dainty [1984] and Menke [1984].

Up until this point, we have discussed only $P$ waves. Some initial simulations have been done involving the propagation of $S V$ waves through random media. Figure 19 depicts $S V$ wave synthetics for the constant velocity case and for an exponential random medium. We consider $S V$ wave pulses with dominant frequencies about half that used for the $P$ waves, so that the wavelength to grid spacing ratio (and hence accuracy) is comparable for the two wave types. The $S V$ waves in the random medium show significant energy on the radial components of displacement and coda after the initial pulse. As with the $P$ waves, the amplitude of the $S V$ wave (transverse component) in the random medium generally decreases with distance relative to the reference case, a result of scattering attenuation. Some energy is observed to arrive before the $S V$ pulse on both the radial and transverse components of the synthetics. This energy is produced by $S V$ to $P$ conversions in the random medium. The initial arrival time of this precursory energy on

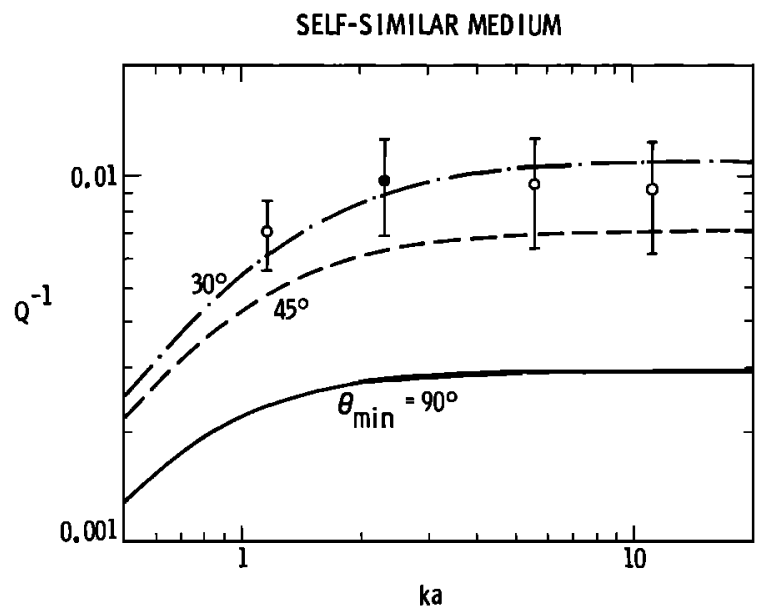

Fig. 18. Measured attenuation and theoretical curves for self-similar random media, $\sigma_{\text {ref }}$ of $10 \%$.
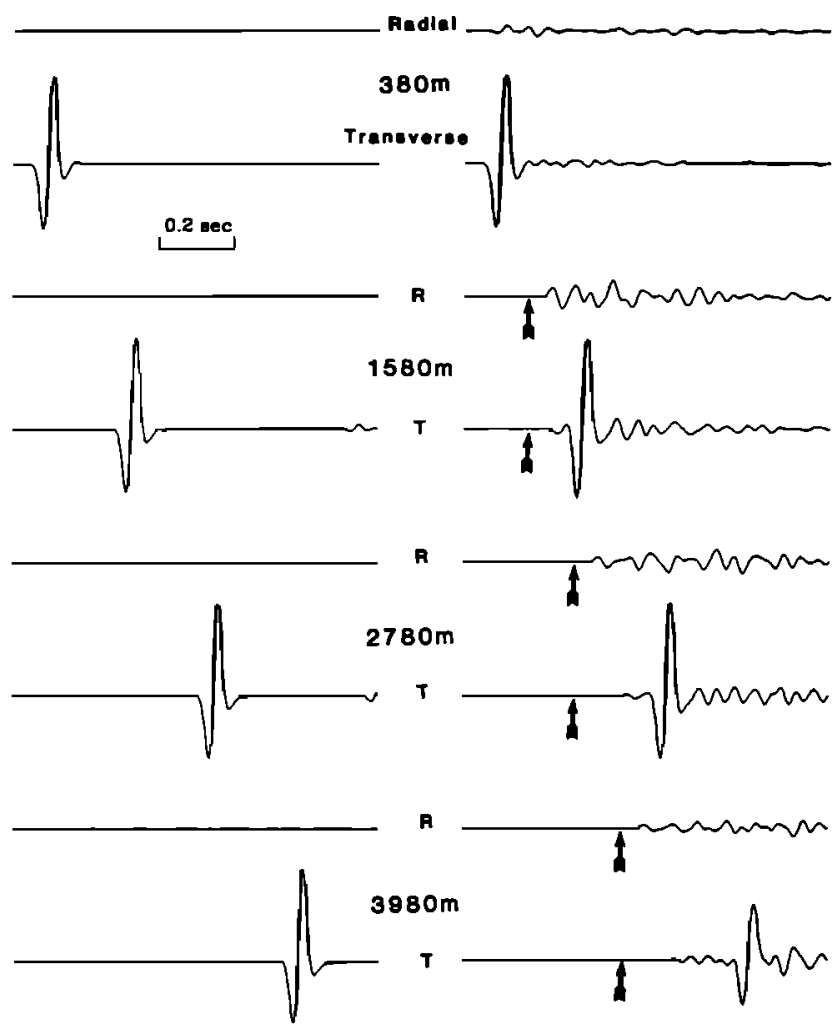

Fig. 19. (Left) Synthetic seismograms for an $S V$ wave in a constant velocity medium (see geometry in Figure 2). (Right) Synthetic seismograms for an $S V$ wave in an exponential random medium with $10 \%$ standard deviation and $a=80 \mathrm{~m}$. Arrows indicate the arrival times calculated for an $S V$ to $P$ conversion occurring in the portion of the random medium nearest the source.

the radial components corresponds approximately to the expected time for such conversions (see Figure 19).

The values of attenuation measured for $S V$ waves in exponential random media are shown in Figure 20 . These values were determined in the same manner as the $P$ waves, except that the waves were band-pass filtered from 12.5 to $17.5 \mathrm{~Hz}$. The frequency dependence of the $S V$ attenuation is similar to

SV WAVE, EXPONENTIAL CORR. FTN.

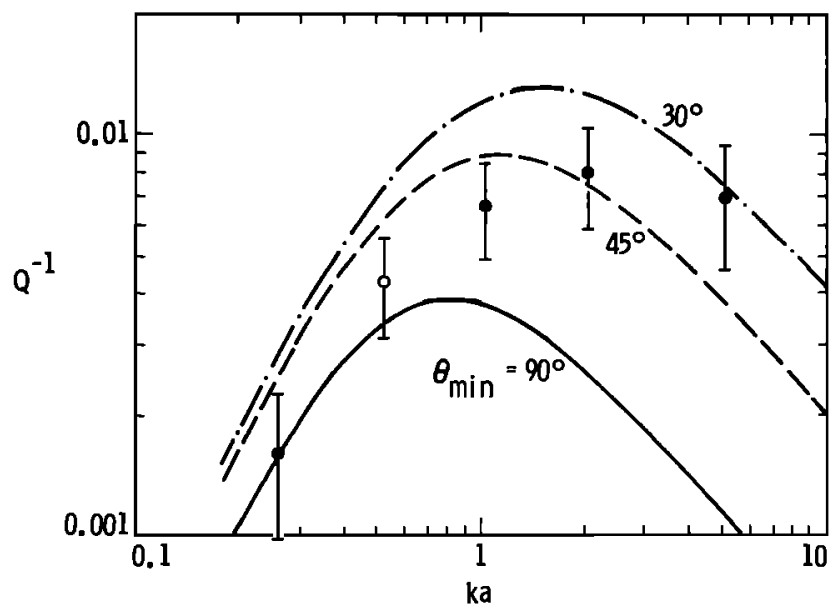

Fig. 20. Measured attenuation and theoretical curves for $S V$ waves in exponential random media $\left(\sigma_{c}=10 \%\right)$. 

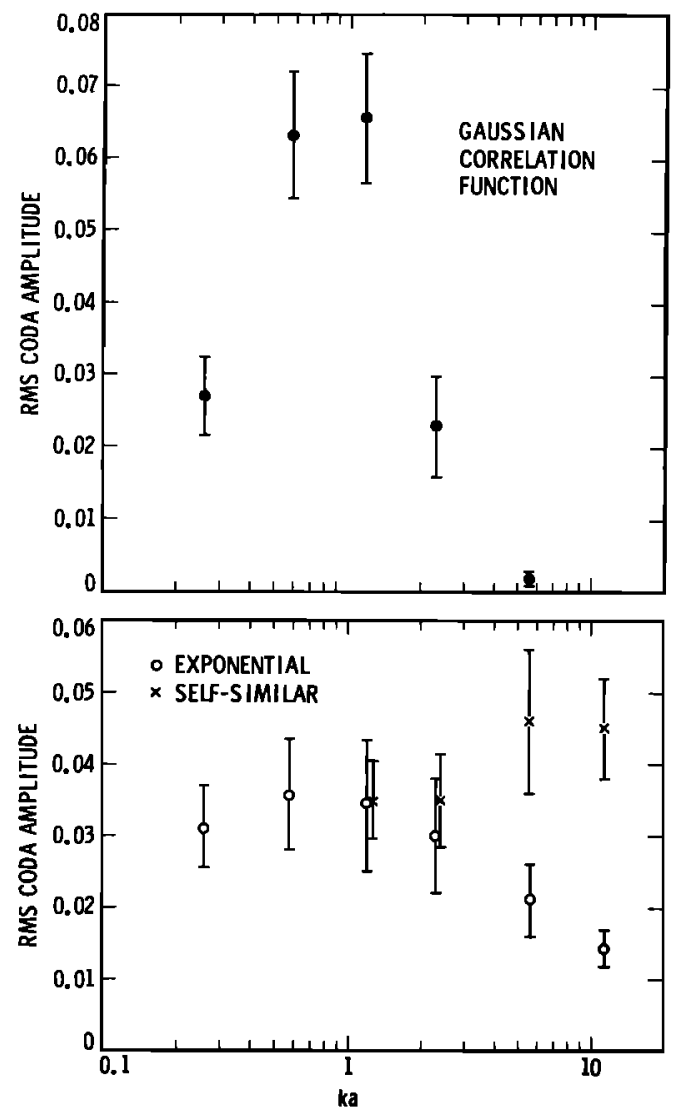

Fig. 21. Root mean square (rms) amplitude of the filtered backscattered coda plotted as a function of $k a$ for Gaussian (top), exponential (bottom), and self-sımilar (bottom) media. Each point represents the average of 5-10 waveforms, with the error bars signifying one standard deviation. Amplitudes are normalized to the peak amplitude of the direct wave.

that observed for $P$ waves in the same media: increase of attenuation with $k a$ for $k a<1$ and a constant level of attenuation for $k a$ between 1 and 6 . These initial results suggest that for $0.5 \leq k a \leq 6$, the amount of attenuation for $S V$ waves is comparable to that of $P$ waves, when waves of equal wavelengths are considered. Variations in Poisson's ratio, which was kept fixed in this study, could of course change this result.

\section{BACKSCATTERED CODA}

\section{Frequency Dependence of Coda Amplitude}

In this section we evaluate the amplitude of the backscattered synthetic coda as a function of $k a$. The backscattered coda was determined from $P$ wave synthetics at receivers located nearest to the source (see Figure 2). The coda waves observed for these receivers consist mainly of energy backscattered from the random medium. The synthetics at each receiver were band-pass filtered $(25-35 \mathrm{~Hz})$ and the root mean square amplitude of the filtered coda was calculated over a fixed time window (0.2-0.6 $\mathrm{s}$ after the $P$ wave arrival time). The results are shown in Figure 21 for Gaussian, exponential, and self-similar random media. Each data point represents the coda amplitude (normalized to the peak amplitude of the direct wave) averaged over 5-10 receivers equidistant from the source. In most cases, the results from two random media (with different seeds) were averaged for each data point.
Figure 21 demonstrates that the dependence of backscattered coda amplitude on $k a$ is generally similar to that of the apparent attenuation, although there is large variance in the coda measurements. For Gaussian media the coda amplitude is greatest at $k a \approx 1$. The coda amplitude for exponential media decreases for $k a>1$ but not as sharply as the Gaussian media. The coda amplitude is approximately constant with respect to $k a$ for the self-similar media. For $k a>1$ the coda amplitudes for the self-similar media are greater than those of the exponential media. The difference in the coda amplitude between the Gaussian, exponential, and self-similar media for $k a>1$ is a result of the difference in the fluctuation amplitude of these media at small wavelengths.

In Figure 22 we plot the observed coda amplitude with respect to the backscattered coda amplitude $\left(\theta=180^{\circ}\right)$ predicted by the acoustic theory (see Appendix C). Although the functional relationship between the coda amplitude and $k a$ can be derived from the theory, the absolute value of the amplitude depends on how the coda waves are attenuated and multiply scattered. The effects of these processes are poorly known at present, so we simply compare the observed coda with relative values from the theory. Dividing equation (C7) by $k$ and setting $\theta=180^{\circ}$, we obtain the backscattered coda energy generated per wavelength travel distance as

$$
A_{c}^{2} \propto k^{2} \sigma^{2} P(2 k)
$$

Figure 22 confirms that the observed coda amplitude is generally proportional to the value predicted by equation (13) for the three types of random media studied.

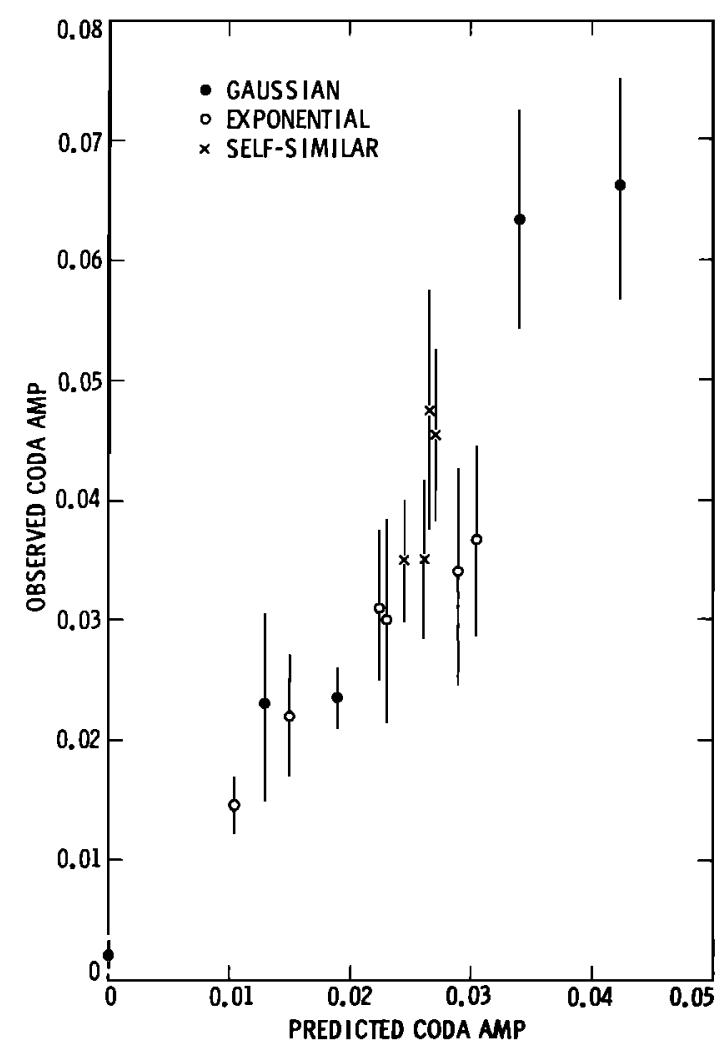

Fig. 22. Observed backscattered coda amplitude plotted against the theoretical amplitude from equation (13). Data and error bars as in Figure 21. The units for the theoretical amplitudes are arbitrary. 


\section{Time Decay of Coda}

The time decay of the seismic coda is an important property of observed high-frequency seismograms that has been studied in detail by numerous investigators. Aki and Chouet [1975] presented a single-scattering model of backscattered coda to explain the coda decay of waveforms from local microearthquakes. This model predicts that the decay rate of the coda is related to the transmission attenuation of the medium. In this portion of the paper we evaluate the time decay of the backscattered coda for the finite difference synthetics and compare the results with the single-scattering theory. For this study we considered acoustic $P$ waves, applying the finite difference scheme to solve the acoustic heterogeneous wave equation (equation (1)). Because of the reduced memory requirements for the acoustic finite difference code relative to the elastic one, larger grid sizes could be utilized. This allows the coda decay rate to be examined over a longer time window without being affected by the original wave front encountering the edges of the grid.

a)

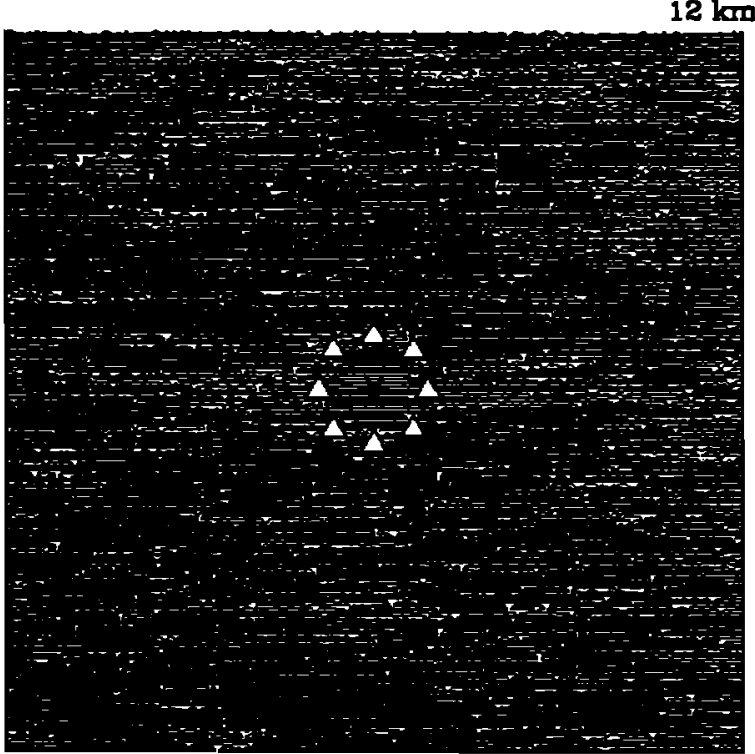

b)

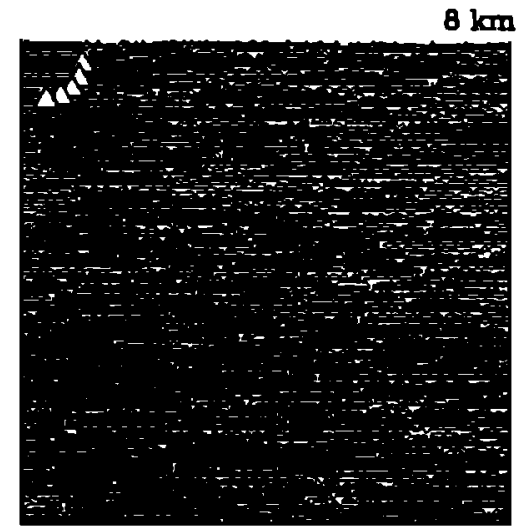

Fig. 23. Configurations of simulations used to study the time decay of the backscattered coda. The source is indicated by the star and the receivers are shown by triangles. All edges have absorbing boundary conditions.

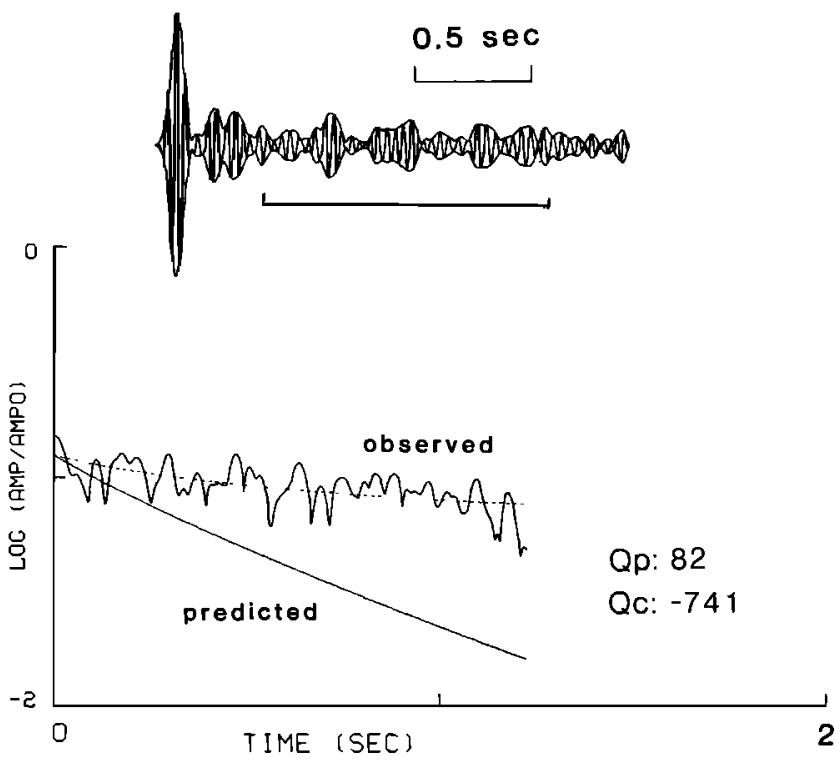

Fig. 24. (Top) Typical synthetic seismogram, filtered between 25 and $35 \mathrm{~Hz}$, derived from the geometry depicted in Figure 23a. The bar indicates the portion of the coda envelope analyzed for the time decay. The medium had a correlation distance of $10 \mathrm{~m}(k a=0.3$ at 30 $\mathrm{Hz}$ ) and a standard deviation of $20 \%$. (Bottom) Average of the log of the coda amplitudes for eight receivers plotted against time, starting $0.45 \mathrm{~s}$ after the direct wave. Amplitudes are normalized to the peak amplitude of the direct pulse. The smooth solid curve is the coda decay predicted by the single-scattering model, derived from the transmission $Q_{p}$ measured for the medium. The dashed curve represents the best fit of the observed coda decay to equation (14). The resulting estimate of the coda $Q_{c}$ is shown to the right.

Two different configurations were used to study the time decay of the backscattered coda and are depicted in Figure 23. For the first simulation (Figure 23a) the source was situated in the center of a 600 by 600 point grid $(12 \mathrm{~km}$ by $12 \mathrm{~km})$, with a set of receivers surrounding it at a distance of 30 grid points $(600 \mathrm{~m})$. Absorbing boundary conditions were invoked at all edges. The random medium had a correlation distance of $10 \mathrm{~m}$ ( $k a=0.3$ at $30 \mathrm{~Hz}$ ), an exponential correlation function, and a standard deviation in velocity of $20 \%$. The synthetic seismograms at each receiver were band-pass filtered $(25-35 \mathrm{~Hz})$ and enveloped (see Figure 24). The log of the envelope amplitude, beginning $0.45 \mathrm{~s}$ after the arrival time of the direct pulse, was averaged over the eight receivers and is shown in Figure 24.

The single-scattering model can be used to predict the coda decay if the transmission $Q_{p}$ of the medium is known. For each random medium described here, the transmission $Q_{p}$ was determined from the amplitude decay of the direct wave with distance. The single-scattering model [Aki and Chouet, 1975] gives the coda amplitude $A(\omega, t)$ at radial frequency $\omega$ and time $t$ for two-dimensional media as

$$
A(\omega, t) \propto\left(\frac{1}{t}\right)^{1 / 2} \exp \left(\frac{-\omega t}{2 Q_{c}}\right)
$$

where $Q_{c}$ is the "coda $Q$ " of the medium. The single-scattering theory equates the coda $Q$ with the transmission $Q$ of the medium, that is, the $Q$ observed for a propagating seismic wave. We compare the observed and predicted coda decay up to the time $\left(t_{\text {edge }}\right)$ corresponding to the travel time from the source to the edge of the grid and back to the receivers. Comparison between the theory and the simulations is inappro- 

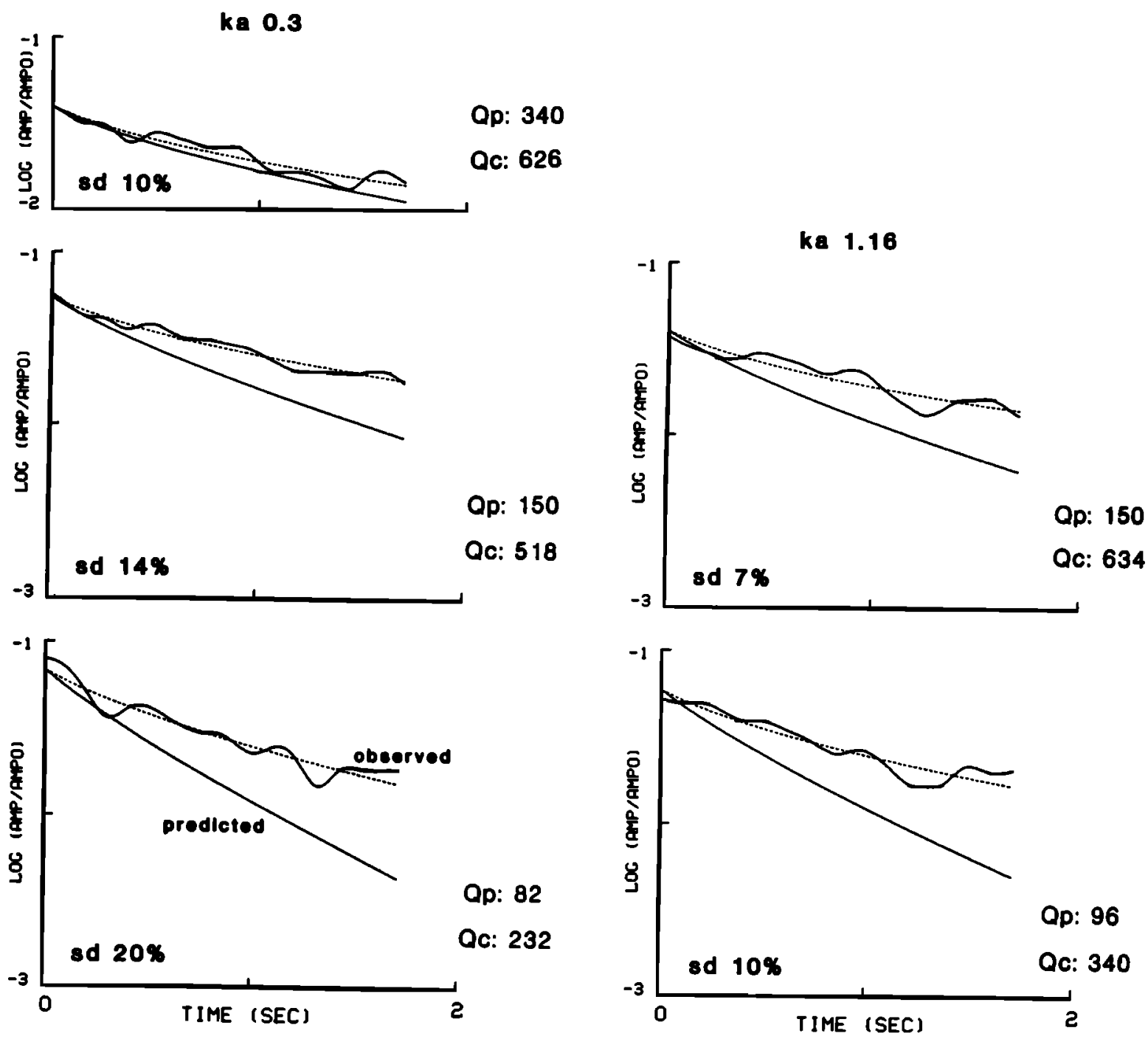

Fig. 25. (Left) Observed and predicted (single-scattering model) coda decays for media with $a=10 \mathrm{~m}(k a=0.3)$ and standard deviations in velocity of 10,14 , and $20 \%$. The coda envelopes have been smoothed with a Gaussian filter (see text). Geometry is shown in Figure $23 \mathrm{~b}$. Coda $Q_{c}$ values derived from the dashed curves and the observed transmission $Q_{p}$ are listed to the right of each panel. (Right) Coda decays for media with $a=40 \mathrm{~m}(\mathrm{k} a=1.16)$ and standard deviations of 7 and $10 \%$

priate for times greater than $t_{\text {edge }}$ because the initial wave front has been absorbed along the edges of the grid.

Figure 24 clearly shows the discrepancy between the coda decay observed in the simulation and that predicted by the single-scattering model. This random medium $(a=10 \mathrm{~m}$, $\sigma=20 \%$ ) has strong scattering attenuation, with a measured $Q_{p}$ of 82 . While the single-scattering theory predicts a steep decay of coda amplitude with time, the observed decay is quite gradual. In fact, fitting equation (14) to the observed coda decay yields a negative value for $Q_{c}$ (see Figure 24). This indicates that the observed coda decay is actually less steep than the geometrical spreading term $t^{-1 / 2}$ in equation (14). The negative value of $Q_{c}$ is a consequence of the inapplicability of the single-scattering theory and equation (14) for this random medium and does not imply a growth of energy with time.

We investigated the coda decay rate for a variety of random media using the geometry shown in Figure $23 b$. This configuration was chosen so that the quarter-circular wave front travels the maximum distance possible in the medium before it encounters the edge of the grid. Absorbing boundary con- ditions are applied at all edges. Since we are only considering one quadrant of the medium, the absolute amplitude of the backscattered coda is about one fourth that for the whole space. We would expect the decay rate of the coda to be similar for the quarter and whole space configurations since the geometrical spreading of the wave front and the increase in the number of scatterers encountered by the wave front with time are identical in both cases. For these simulations we considered random media with the same correlation distance but differing standard deviations in velocity. The transmission attenuation $\left(Q_{p}{ }^{-1}\right)$ measured for these media was found to be approximately proportional to the variance of the velocity [Frankel and Clayton, 1984]. For each medium the log of the coda envelope at each receiver was smoothed in the time domain using a Gaussian filter with a width of about $0.2 \mathrm{~s}$ at half amplitude. The smoothed coda envelopes were then averaged over the five receivers and plotted up to time $t_{\text {edge }}$ for this configuration.

The observed coda decays for three exponential random media with $k a=0.3$ but differing standard deviations in veloc- 
ity $(10,14$, and $20 \%)$ are depicted on the left side of Figure 25. For the medium with a standard deviation of $10 \%$, the predicted coda decay appears to agree with the observations from the synthetics. In this case, the transmission $Q_{p}$ is relatively high, 340 , and the scattering is therefore weak. The predicted coda decay does not match the synthetic results, however, when the scattering attenuation is moderate. The observed coda decay is more gradual than the theoretical one for the medium with a $14 \%$ standard deviation in velocity and a $Q_{p}$ of 150 . The difference between the observed and predicted decay is most striking for the medium with $20 \%$ standard deviation and a $Q_{p}$ of 82 . As in Figure 24, the theory predicts a sharp rate of coda decay, whereas the observed coda decay is quite gradual, similar to the decay rates of the more weakly scattering media.

Simulations using media with a larger correlation distance confirm that the amount of the scattering attenuation is the critical factor that causes the difference between the observed coda decay and that predicted by the single-scattering theory. On the right side of Figure 25, we show that observed coda decay for two exponential media with $a=40 \mathrm{~m}(\mathrm{ka}=1.16$ at $30 \mathrm{~Hz}$ ) and standard deviations of 7 and $10 \%$. The values of $Q_{p}$ determined for these media were $150(\sigma=7 \%)$ and 96 $(\sigma=10 \%)$. Again the observed coda decay rates are more gradual than predicted by the single-scattering theory, with the difference increasing as the transmission attenuation increases.

The simulations indicate that estimates of coda $Q$ derived from the coda decay using the single-scattering model will significantly overestimate the transmission $Q_{p}$ of the medium, when $Q_{p} \leq 200$. In Figure 25 we fit the observed coda decays to equation (14) and obtained values of the coda $Q$. The ratio between $Q_{c}$ and $Q_{p}$ is about three for $Q_{p} \leq 150$. The calculated coda $Q$ decreases somewhat as $Q_{p}$ decreases, signifying that the coda decay rate steepens slightly. This dependence of the coda decay on the transmission attenuation is opposite to that observed for forward scattered coda in randomly layered media by Menke and Chen [1984]. They reported that the decay rate of the forward scattered coda decreases as the variance of the medium (and hence its transmission attenuation) increases.

The discrepancy between the observed and predicted coda decay appears to be caused by multiple scattering. As the scattering attenuation gets stronger, multiple scattering becomes more important, and the coda decay becomes more gradual than predicted by the single-scattering theory. In numerical studies of one-dimensional random media, Richards and Menke [1983] also found that multiple scattering produced more gradual decays of the backscattered coda relative to the single-scattering model. The results obtained here indicate that multiply scattered waves form a significant portion of the backscattered coda when the scattering attenuation is greater than a certain threshold $\left(Q_{p} \leq 200\right)$.

Gao et al. [1983] extended the Aki and Chouet [1975] theory to include multiple scattering, while still neglecting losses in transmission (Born approximation). This theory predicts a coda decay rate more gradual than found from the single-scattering model for large lapse times. Gao et al. [1983] conclude that multiple scattering would cause estimates of coda $Q$ derived from the single-scattering model to be 1.4 times that of the transmission $Q$ of the medium. However, the simulations demonstrate that multiple scattering can alter the coda decay in a much stronger manner, such that the calcu- lated coda $Q$ can actually be negative when the scattering attenuation is large (Figure 24).

\section{Discussion}

The results of the numerical simulations indicate that a crust with self-similar variations in seismic velocity over length scales of less than about $50 \mathrm{~km}(a \geq 10 \mathrm{~km})$ can explain a wide variety of observations from actual short-period seismograms. This self-similar model of the crust is consistent with general observations concerning rocks and surface geology. The seismic velocity within a rock can vary markedly over length scales of millimeters, as crystals of differing minerals are sampled. Comparable variations in seismic velocity exist in the crust over length scales of up to tens of kilometers (at least) and are manifested by differences in the average velocities of distinct lithologic units.

The self-similar model contrasts with that proposed by $A k i$ $[1980 a, b]$, who concluded that the crust was characterized by a correlation distance of about $1 \mathrm{~km}$. Such a model could account for a peak of shear wave attenuation at $1 \mathrm{~Hz}$ inferred by $A k i[1980 a]$, based on long-period surface wave measurements and studies of $S$ and coda waves from microearthquakes. To explain the apparent frequency dependence of $Q$ above $1 \mathrm{~Hz}, A k i[1980 \mathrm{~b}]$ suggested that the spectrum of crustal velocity variations was intermediate between that of exponential and self-similar media for scale lengths less than a few kilometers. Studies of coda $Q$ generally report a strong increase of $Q_{c}$ with frequency between 1 and $25 \mathrm{~Hz}$ [ $A k i$ and Chouet, 1975; Rautian and Khalturin, 1978; Roecker et al., 1982]. However, the results of the numerical study described in this paper clearly show that the single-scattering model used for these $Q$ estimates is not appropriate when the scattering $Q$ is moderate. Thus the coda $Q$ results may sometimes overestimate the body wave $Q$.

Hermann [1980] reported a regional correspondence between $Q_{c}$ and transmission $Q$ for $1-\mathrm{Hz} L_{g}$ waves. He found a $Q_{c}$ of about 140 using records from Berkeley, California, which agreed roughly with measurements of transmission $Q$ in the area made by other investigators. If this attenuation was caused by scattering, it would imply fairly strong scattering and the presence of multiply scattered waves in the coda. Given the results of the numerical simulations, we would not expect a correspondence between coda $Q$ and transmission $Q$ in media with such strong scattering. This agreement would be expected, however, if intrinsic loss mechanisms dominated the attenuation for $1-\mathrm{Hz} L_{g}$ waves in central California. When the observed transmission attenuation is large, the similarity between the coda $Q$ and the transmission $Q$ may imply that intrinsic absorption is the cause of the attenuation. Conversely, the presence of a relatively gradual rate of coda decay in a medium with strong transmission attenuation may indicate that the attenuation is produced by scattering rather than intrinsic dissipation.

The characterization of the frequency dependence of crustal attenuation at frequencies greater than about $1 \mathrm{~Hz}$ would discriminate between the self-similar and exponential models of crustal heterogeneity if attenuation is indeed caused by scattering in this frequency range. For these frequencies the scattering $Q$ of the self-similar medium proposed here $(a \geq 10$ $\mathrm{km}$ ) would be constant with frequency and that of an exponential medium $(a=1 \mathrm{~km})$ would linearly increase with frequency. $A k i[1980 a]$ estimated the frequency dependence of $Q_{s}$ in this frequency range from the amplitudes of earthquakes 
at varying distances from a single station, recorded through real-time band-pass filters. The amplitudes were corrected for the source strength by dividing by the amplitude of the seismic coda observed at some fixed time after the origin time. This "single-station" method documented that $Q \propto f^{0.6-0.8}$ between 1.5 and $24 \mathrm{~Hz}$ in Japan. Although we feel that the assumptions invoked in this method are reasonable, $Q$ measurements derived from changes in body wave spectra as a function of source-receiver distance would be useful as a comparison.

Improved determinations of transmission attenuation using direct spectral methods are needed to resolve the frequency dependence of attenuation from 1 to $30 \mathrm{~Hz}$. If $Q$ is proportional to frequency, the spectral shape will not change as the wave propagates, only the overall amplitude will decrease. Anderson and Hough [1984] noted that the shape of the high-frequency spectral falloff for strong motion records was consistent with a frequency independent $Q$. There is some evidence of a constant $Q$ with frequency from 1 to $16 \mathrm{~Hz}$ in the amplitudes of filtered strong motion records from the San Fernando earthquake [Berrill, 1975; Hanks, 1982]. Singh et al. [1982], however, reported an increase of $Q$ with frequency using spectra from some Imperial Valley aftershocks.

While this paper attempts to explain the features of shortperiod seismograms with an isotropic random medium model, the earth's crust is undoubtedly even more complex. The presence of fine scale horizontal layering can produce seismic coda (see, e.g., Bouchon [1982]) and apparent attenuation [Richards and Menke, 1983]. Levander and Hill [1985] have used finite difference simulations to demonstrate that random topography on buried interfaces can also generate coda waves. The heterogeneity spectrum of the crust may also change with depth, particularly in the near surface where open joints and fractures are present. Studies of high-frequency spectra ( $>10$ $\mathrm{Hz}$ ) indicate that severe attenuation often occurs at shallow depths (less than a few kilometers) in the earth's crust, attenuation much greater than that observed for ray paths that primarily sample depths larger than $5 \mathrm{~km}$ [Frankel, 1982b; Hanks, 1982; Malin and Waller, 1985; Cranswick et al., 1985]. If this "site response" is caused by scattering from near-surface heterogeneities, it would imply that the small-scale velocity fluctuations ( $<100 \mathrm{~m}$ across) would be larger in amplitude near the surface than at deeper depths in the crust.

It is clear that the complete quantification of the various scattering effects discussed in this paper will require substantial future effort, in both the use of numerical simulations and the analysis of actual short-period seismograms. As more powerful computers become available, particularly those that use concurrent processing architecture (Appendix A), finite difference simulations in large-scale, three-dimensional random media will become feasible. We feel that the major conclusions of this paper are not affected by the two dimensionality of the simulations. The peak values of scattering attenuation differ little between the two- and three-dimensional theories. We would expect that the coda amplitude would also be similar for the two cases, with the three-dimensional medium having more scatterers per travel distance but a stronger rate of geometrical spreading. In any case, even factor of 2 variations in coda amplitudes would not alter the principal findings of the paper.

\section{Conclusions}

1. The earth's crust must have a correlation distance of at least $10 \mathrm{~km}$ to account for the standard deviation and spatial correlation of travel time variations observed for large-scale seismic arrays.

2. Exponential and Gaussian random media with correlation distances of $10 \mathrm{~km}$ and larger ( $\leq 10 \%$ standard deviation in velocity) cannot account for the presence of seismic coda at frequencies of $30 \mathrm{~Hz}$, commonly observed for microearthquakes.

3. A random medium with self-similar velocity fluctuations ( $a \geq 10 \mathrm{~km}$ and standard deviation of about $5 \%$ ) can explain the observations of both travel time variations and high-frequency coda amplitudes.

4. The scattering attenuation $\left(Q^{-1}\right)$ observed in the simulations increases with $k a$ for $k a<1$ for Gaussian and exponential media. The scattering attenuation is peaked at $k a \approx 1$ for Gaussian media. The apparent attenuation in exponential and self-similar media appears to be constant for $1 \leq k a \leq 5.6$.

5. The single-scattering model of coda formation is not valid when the scattering attenuation is moderate or large (scattering $Q \leq 200$ ). Because of multiple scattering the coda decay rates observed in the numerical simulations are relatively gradual when the scattering attenuation is large.

\section{APPENDIX A: Accuracy Tests of Finite DiFFERENCE METHOD}

The complexity of the random media and the resulting synthetic seismograms naturally raises questions about the accuracy of the finite difference method. We have performed a series of tests to evaluate the accuracy of the fourth-order schemes used in this paper. Frankel and Clayton [1984] computed the finite difference synthetics for a plane wave propagating at normal incidence through a stack of plane layers with randomly varying velocities. Comparison of the finite difference synthetics with those derived from the Haskell [1960] matrix method showed excellent agreement.

A second accuracy test involved the evaluation of the radiation pattern from a point scatterer from an incident plane wave. Figure A1 illustrates the radiation pattern observed with the finite difference synthetics for $S V$ waves scattered from a small scatterer for an incident plane $S V$ wave. The

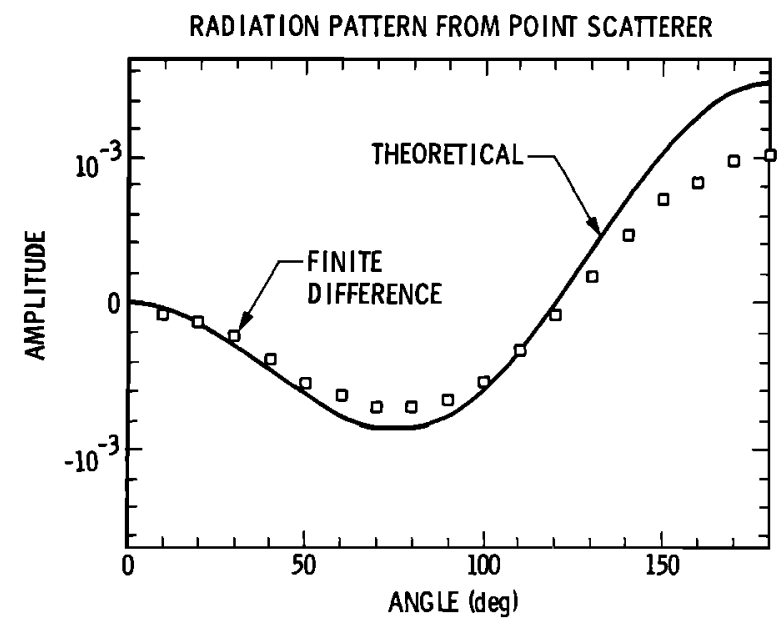

Fig. A1. Radiation pattern observed in the finite difference simulations for $S V$ waves scattered from a small scatterer, for an incident plane $S V$ wave. The theoretical curve is derived from the analytical results of $W u$ and $A k i$ [1985], adapted to the two-dimensional geometry of the simulations. 
scatterer was 2 grid points on a side and was characterized by an impedance contrast of $1 \%$ relative to the surrounding medium but with the same $P$ and $S$ wave velocities. The wavelength of the incident wave was 10 grid points. The radiation pattern was measured from the amplitude of the curl of the scattered wave field. The radiation pattern derived from the synthetics shows good correlation (Figure A1) with that predicted by the analytical theory of $W u$ and $A k i$ [1985], modified to the two-dimensional geometry of the simulations. The features that differ between the two- and three-dimensional cases are the Green's functions and the substitution of scatterer area for volume (see Appendix C). In the twodimensional geometry the scatterer is essentially a line scatterer extending perpendicular to the plane of the grid. The largest discrepancy in the absolute amplitude of the synthetics and the theory occurs for the backscattered energy $\left(\theta=180^{\circ}\right)$, where the amplitude of the synthetics is about two thirds of the predicted value. This difference is not surprising, given the finite extent of the scatterer and the fact that sharp contrasts in material parameters are represented as gradients in the finite difference scheme.
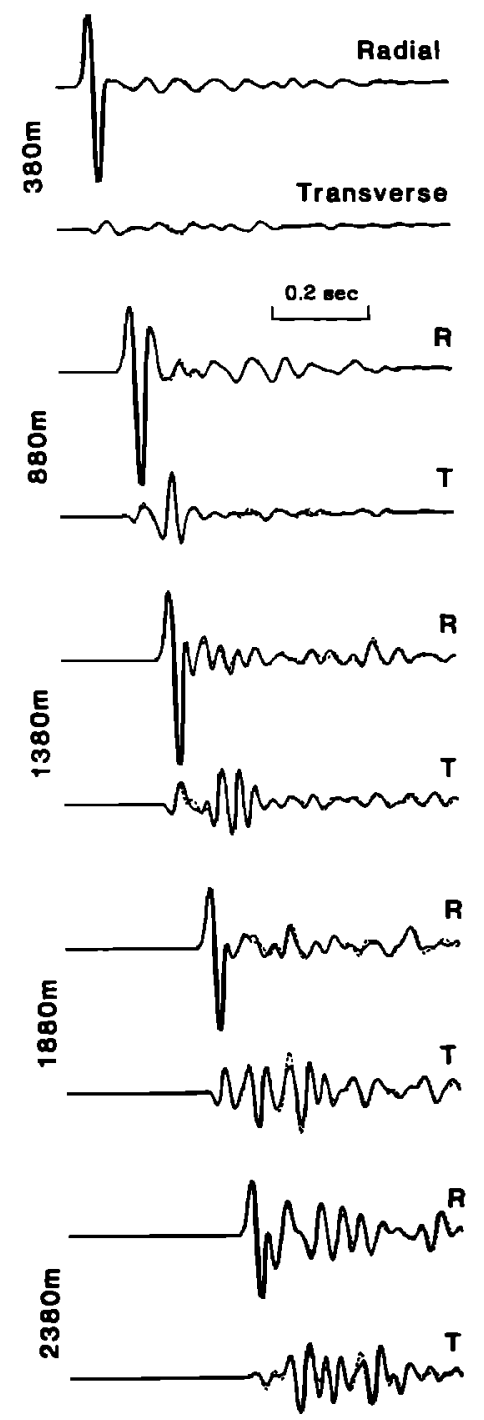

Fig. A2. Comparison of synthetic seismograms made in the simulations using two different grid spacings. Seismograms with solid lines were generated with a grid spacing of $20 \mathrm{~m}$, and those with dashed lines were produced with a grid spacing of $10 \mathrm{~m}$, in the same random medium. Source-receiver distances are shown to the left.
Our third accuracy test consisted of running simulations for the same random medium at two different grid spacings. Synthetic seismograms were derived for a random medium with a grid spacing of $20 \mathrm{~m}$. The random medium was then interpolated to yield a grid spacing of $10 \mathrm{~m}$, with twice the number of grid points in each direction ( $x$ and $z$ ). The resulting synthetics were then compared with the original run. Any numerical errors will cause discrepancies between the synthetics derived from the two cases. Figure A2 depicts some of the seismograms used in this comparison. Although minor differences are apparent in some of the transverse components, the overall good agreement between the waveforms indicates that numerical errors are not significantly affecting the results. In this paper we analyze synthetic $P$ waves with wavelengths at least 10 times the grid spacing.

Some of the finite difference simulations described in this paper (coda time decay) were run on one of the concurrent processors developed and built by the Jet Propulsion Laboratory and the California Institute of Technology. This concurrent processor consists of 32 individual processors (nodes) which perform their operations simultaneously and can communicate with their adjacent processors. For the twodimensional finite difference scheme considered here, the finite difference grid is divided into 32 equal-sized subgrids. Each separate processor solves the finite difference algorithm in a particular subgrid. The processors only communicate with each other for the exchange of updated values along the common edges of the subgrids. This communication occurs only once per time step and takes relatively little time compared to the computations for the interior of the subgrids. Thus problems on large grids can be solved in virtually the time it takes each processor to solve its small subgrid. The concurrent processing technique will make the solution of large-scale, two- and three-dimensional problems in wave propagation possible at substantial savings of cost and computing time, relative to sequential computers.

\section{APpendix B: Normalization of Discrete RANDOM MEDIA}

The finite grid spacing introduces, in some cases, certain differences in the normalization of the random medium, compared to the case of a continuous medium. For a continuous medium, the variance $\left(\sigma_{c}{ }^{2}\right)$ is given by

$$
\sigma_{\mathrm{c}}{ }^{2}=\int_{0}^{\infty} P\left(k_{\mathrm{r}}\right) k_{\mathrm{r}} d k_{\mathrm{r}}
$$

The variance for the same medium sampled on a discrete grid $\left(\sigma_{d}^{2}\right)$ is, however,

$$
\sigma_{d}^{2}=\int_{0}^{k_{\mathrm{Nyq}}} P\left(k_{r}\right) k_{r} d k_{r}
$$

where $k_{\mathrm{Nyq}}$ is the Nyquist wave number $(\pi / h$, where $h$ is the grid spacing). Thus the standard deviation of the discretely sampled medium may be less than that of the original, continuous medium. In the spatial domain this is equivalent to truncating the correlation function at zero offset. In cases where the power spectrum has significant amplitude at the Nyquist wave number, the standard deviation will be a function of the grid spacing. We have normalized the discrete random media to correspond to continuous media with a given $\sigma_{c}^{2}$. 
TABLE B1. Standard Deviations $\sigma_{d}$ of Discrete Random Media Used in Simulations (for $\sigma_{c}=10 \%$ )

\begin{tabular}{lccc}
\hline \multicolumn{1}{c}{ Medium } & $k a$ & $a, \mathrm{~m}$ & $\sigma_{d}, \%$ \\
\hline Gaussian & 0.29 & 10 & 6.8 \\
Gaussian & 0.58 & 20 & 9.6 \\
Exponential & 0.29 & 10 & 6.8 \\
Exponential & 0.58 & 20 & 8.4 \\
Exponential & 1.16 & 40 & 9.2 \\
Exponential & 2.32 & 80 & 9.6 \\
Self-similar & 1.16 & 40 & 7.3 \\
Self-similar & 2.32 & 80 & 8.6 \\
\hline
\end{tabular}

\section{Gaussian and Exponential Media}

In the section on apparent attenuation, we measured the attenuation as a function of $k a$ by studying media with different correlation distances. For a Gaussian correlation function, the two-dimensional fluctuation power spectrum for each of these media is specified by (see Table 1)

$$
P\left(k_{r}\right)=\frac{\sigma_{c}^{2} a^{2}}{2} e^{-k_{r}^{2} a^{2} / 4}
$$

where $\sigma_{c}{ }^{2}$ is the variance of the continuous medium. From Table 1 and equation (B2) the variance of the discrete Gaussian media used in the simulations can be calculated as

$$
\begin{gathered}
\sigma_{d}^{2}=\int_{0}^{k_{\mathrm{N} y \mathrm{q}}} \frac{\sigma_{c}{ }^{2} a^{2}}{2} e^{-k_{r}{ }^{2} a^{2} / 4} k_{r} d k_{r} \\
\sigma_{d}{ }^{2}=\sigma_{c}{ }^{2}\left(1-e^{\left.-k_{\mathrm{Nyq}^{2} a^{2} / 4}\right)}\right.
\end{gathered}
$$

For exponential media the spectral power and discrete variance are

$$
\begin{gathered}
P\left(k_{r}\right)=\sigma_{c}{ }^{2} a^{2}\left(1+k_{r}{ }^{2} a^{2}\right)^{-3 / 2} \\
{\sigma_{d}}^{2}=\sigma_{c}{ }^{2}\left[1-\left(1+k_{\mathrm{Nyq}}{ }^{2} a^{2}\right)^{-1 / 2}\right]
\end{gathered}
$$

Using equations (B5) and (B7), it was found that the standard deviation of the discrete media differed significantly from that of the continuous medium when $a<2 h$. The values of the standard deviations of these discrete media used in the simulations are listed in Table B1.

\section{Self-Similar Media}

The discrete, self-similar media used in this paper cannot be normalized with respect to the standard deviation of the continuous medium because that standard deviation is undefined. That is, the correlation function $K_{\mathrm{o}}(r / a)$ is infinite at $r=0$. Our approach is to use the standard deviation for a particular, discrete, self-similar medium as the reference by which the other self-similar media are normalized. Fortunately, the variance of the discrete self-similar medium is a logarithmic function of the grid spacing, so that large differences in the sampling interval do not strongly alter the determinations of the standard deviation of the medium. This can be seen from the variance $\sigma_{\text {ref }}^{2}$ for a reference medium with $a=a_{\text {ref }}$

$$
\begin{gathered}
\sigma_{\mathrm{ref}}^{2}=C \int_{0}^{k_{\mathrm{Nyq}}} a_{\mathrm{ref}}{ }^{2}\left(1+k_{\mathrm{r}}^{2} a_{\mathrm{ref}}{ }^{2}\right)^{-1} k_{\mathrm{r}} d k_{\mathrm{r}} \\
\sigma_{\mathrm{ref}}^{2}=\frac{C}{2} \ln \left(1+k_{\mathrm{Nyq}}{ }^{2} a_{\mathrm{ref}}{ }^{2}\right) \\
\sigma_{\mathrm{ref}}^{2}=\frac{C}{2} \ln \left(1+\frac{\pi^{2} a_{\mathrm{ref}}{ }^{2}}{h^{2}}\right)
\end{gathered}
$$

$C$ is a constant that specifies the absolute amplitude of the power spectrum for a given $\sigma_{\text {ref }}{ }^{2}$ and can be found from equation (B9):

$$
C=\frac{2 \sigma_{\mathrm{ref}}^{2}}{\ln \left(1+k_{\mathrm{Nyq}}{ }^{2} a_{\mathrm{ref}}^{2}\right)}=0.29{\sigma_{\mathrm{ref}}}^{2}
$$

Here, a self-similar medium with $a=200 \mathrm{~m}$ and $h=20 \mathrm{~m}$ was chosen as the reference medium. Thus the two-dimensional fluctuation power spectrum for the self-similar media used in our investigation of apparent attenuation is given by

$$
P\left(k_{r}\right)=0.29 \sigma_{\text {ref }}^{2} a^{2}\left(1+k_{r}^{2} a^{2}\right)^{-1}
$$

The dependence of apparent attenuation on $k a$ in self-similar media was quantified with the finite difference simulations by considering media with differing $a$ but whose spectra are described by equation (B12). The variance $\sigma_{d}^{2}$ of each medium is

$$
\sigma_{d}^{2}=\int_{0}^{k_{\mathrm{Nyq}}} 0.29 \sigma_{\mathrm{ref}}{ }^{2} a^{2}\left(1+k_{r}{ }^{2} a^{2}\right)^{-1} k_{r} d k_{r}
$$

and

$$
\sigma_{d}^{2}=0.145 \sigma_{\text {ref }}^{2} \ln \left(1+k_{\mathrm{Nyq}}^{2} a^{2}\right)
$$

Thus the standard deviations of such self-similar media vary somewhat as a function of the correlation distance. Table B1 lists the standard deviations used to normalize the self-similar media for the cases that differed significantly from the $10 \%$ standard deviation for the reference medium with $a=200 \mathrm{~m}$. These standard deviations were calculated from equation (B14).

The correlation distance $a$ of a self-similar medium does not correspond to the spatial lag where the amplitude of the correlation function equals $1 / e$, as with the Gaussian and exponential media. Instead, the correlation distance of the selfsimilar medium (and the other types of media) describes the shape of its fluctuation spectrum, specifically its corner wave number.

\section{Discrete Random Media With Correlation Distances Larger Than the Grid Dimension}

In the section of this paper entitled "coda amplitude at high frequencies," several random media were investigated that had correlation distances larger than the total grid size. It was necessary to normalize these media by their spectral amplitude because the standard deviation will be dependent on the total grid size. Therefore the media were constructed differently from those used to quantify the apparent attenuation and travel time. The random media were generated in the wave number domain by assigning the spectral power at each $\left(k_{x}, k_{z}\right)$ to that of the desired correlation function. The phase at each $\left(k_{x}, k_{z}\right)$ was chosen randomly. The resulting wave number spectrum was inversely transformed to the spatial domain to produce the random medium.

Normalization of these media was a two-step process, involving grids with two different mesh spacings. First, a grid spacing of $500 \mathrm{~m}$ and a total grid length of $100 \mathrm{~km}$ was used to make media with correlation distances of greater than 5 $\mathrm{km}$. These media were normalized by their standard deviation, and the spectral amplitude at a particular wave number $\left(k_{\text {norm }}\right)$ was calculated. Then a random medium was constructed with a grid size of $20 \mathrm{~m}$ and total length of $4 \mathrm{~km}$. This grid was used for the simulations of high-frequency $(>10 \mathrm{~Hz})$ wave propagation. The spectral amplitude at $k_{\text {norm }}$ was adjusted to match that obtained from the larger grid. 
The standard deviation increases with total grid size for exponential media where the correlation distance is greater than the grid size. Thus all standard deviations for this section of the paper refer to the standard deviations derived from the grid with $h=500 \mathrm{~m}$. The standard deviation observed on the smaller grid $(h=20 \mathrm{~m})$ is about 0.3 times that of the larger grid for $a=10 \mathrm{~km}$. For self-similar media, however, the variances calculated on the two grids are approximately equal.

\section{APPENDIX C: ThEORETICAL Formulas for APPARENT AtTENUATION IN Two-Dimensional RANDOM MEDIA}

Here we adapt the theory presented by Chernov [1960] and $W u$ [1982] for the scattering of scalar waves through threedimensional random media to the two-dimensional geometry of the finite difference simulations. The derivation of the formula relating the scattering attenuation of a random medium to its fluctuation spectrum consists of two steps. First, the intensity of energy scattered at a particular angle is found for an incident plane wave. Next, the scattered energy is integrated over a range of scattering angles to determine the total apparent attenuation of the random medium. Several assumptions are made in this treatment, including (1) single scattering, (2) weak velocity perturbations, (3) no scattering from gradients in velocity, and (4) receiver located in far-field from scattering region.

We determine the amplitude of waves scattered through angle $\theta$ for an incident plane wave in a two-dimensional random medium, following closely the procedure of $A k i$ and Richards [1980, chapter 13] for the three-dimensional case. Figure $\mathrm{Cl}$ shows the geometry of the problem. For a receiver located at $\mathbf{x}$, the amplitude of a wave (wave number $k$, radial frequency $\omega$ ) scattered by a unit area of the random medium is

$$
\Phi(\mathbf{x}, t)=\Phi_{0}\left(2 \omega^{2} \frac{\delta c}{c}\right)\left[\frac{i}{4 c^{2}} H_{0}^{(2)}(k r)\right] e^{i \omega t} e^{-i k \xi_{1}}
$$

Here $\Phi_{0}$ is the amplitude of the incident plane wave traveling a distance of $\xi_{1}$ from the center of the heterogeneous region to the unit scatterer. $\delta c / c$ is the velocity perturbation, $r$ is the distance between the scattering element and the receiver, and $H_{0}{ }^{(2)}(k r)$ is a Hankel function. The first term in parentheses in the right-hand side of equation (C1) describes the scattering potential and the term in brackets represents the Green's function for a line scatterer or source (perpendicular to the plane) in a two-dimensional medium [see Kraut, 1967]. The far-field approximation allows us to replace $r$ with $|\mathbf{x}|-\mathbf{n} \cdot \boldsymbol{\xi}$, where $\mathbf{n}$ is the unit vector pointing along $\mathbf{x}$. Integrating over the area $A$ of the heterogeneous region and replacing the Hankel function by its asymptotic limit, we obtain

$$
\begin{aligned}
& \Phi=\Phi_{0} \frac{k^{3 / 2}}{2}\left(\frac{2}{\pi|\mathbf{x}|}\right)^{1 / 2} \exp [i(\omega t+\pi / 4-k|\mathbf{x}|)] \\
& \cdot \int_{A} i \sigma(\xi) \exp \left[-i k\left(\xi_{1}-\mathbf{n} \cdot \xi\right)\right] d A
\end{aligned}
$$

where $\sigma(\xi)=\delta c / c$. The spectral power of the scattered wave is determined from the product of the right hand side of equation $(\mathrm{C} 2)$ evaluated at $\xi$ and its complex conjugate at location $\xi^{\prime}$, so that

$$
\begin{aligned}
\left(\frac{\Phi}{\Phi_{0}}\right)^{2} & =\frac{k^{3}}{2 \pi|\mathbf{x}|} \int_{A} \int_{A}\left\langle\sigma\left(\xi^{\prime}\right) \sigma(\xi)\right\rangle \\
& \cdot \exp \left[i k\left(\xi_{1}-\xi_{1}{ }^{\prime}-\mathbf{n} \cdot\left(\xi-\xi^{\prime}\right)\right] d A(\xi) d A\left(\xi^{\prime}\right)\right.
\end{aligned}
$$

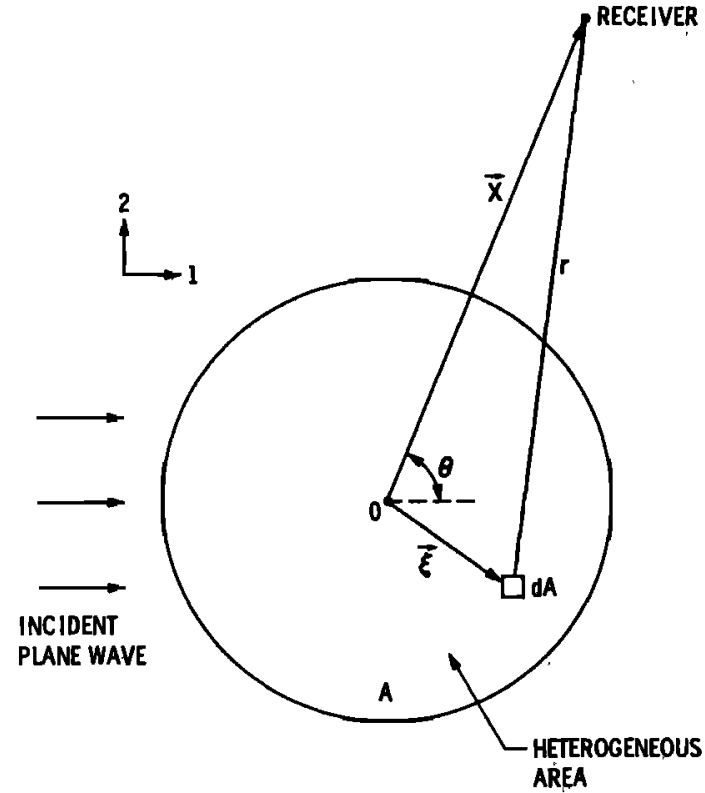

Fig. C1. Geometry used in derivation of formulas for apparent attenuation in random media (modified from $A k i$ and Richards [1980]).

$\left\langle\sigma\left(\xi^{\prime}\right) \sigma(\xi)\right\rangle$ represents an ensemble average of the velocity fluctuations of the medium. We define $K=\mathbf{e}_{1}-\mathbf{n}$, where $\mathbf{e}_{1}$ is the unit vector in the $\xi_{1}$ direction. It can be shown [Aki and Richards, 1980] that $|\mathbf{K}|=2 \sin (\theta / 2)$, where $\theta$ is the angle between the incident and scattered wave. After replacing $\left\langle\sigma\left(\xi^{\prime}\right) \sigma(\xi)\right\rangle$ by $\sigma^{2} N\left(\xi-\xi^{\prime}\right)$, equation (C3) becomes

$$
\begin{aligned}
&\left(\frac{\Phi}{\Phi_{0}}\right)^{2}=\frac{k^{3} \sigma^{2}}{2 \pi|\mathbf{x}|} \int_{A} \int_{A} N\left(\xi-\xi^{\prime}\right) \\
& \cdot \exp \left[i k \mathbf{K} \cdot\left(\xi-\xi^{\prime}\right)\right] d A(\xi) d A\left(\xi^{\prime}\right)
\end{aligned}
$$

Adopting center of mass coordinates $\xi^{c}=\left(\xi+\xi^{\prime}\right) / 2$ and $\xi^{d}=$ $\xi-\xi^{\prime}$, and changing $\left(\xi_{1}^{d}, \xi_{2}^{d}\right)$ to circular coordinates $\left(r^{\prime}, \theta^{\prime}\right)$, the following relationships are found:

$$
\begin{gathered}
\int_{A} d \xi_{1}{ }^{c} d \xi_{2}{ }^{c}=A \\
r^{\prime}=\left|\xi^{d}\right| \\
\mathbf{K} \cdot \xi^{d}=|\mathbf{K}| r^{\prime} \cos \theta^{\prime}=2 \sin (\theta / 2) r^{\prime} \cos \theta^{\prime} \\
d A\left(\xi^{d}\right)=r^{\prime} d r^{\prime} d \theta^{\prime}
\end{gathered}
$$

Substitution of these terms into equation (C4) yields

$$
\begin{aligned}
\left(\frac{\Phi}{\Phi_{0}}\right)^{2}=\frac{k^{3} \sigma^{2} A}{2 \pi|\mathbf{x}|} \int_{r^{\prime}}=0 \quad \int_{\theta=-\pi}^{r^{\prime}=\infty} N\left(r^{\prime}\right) & \\
& \cdot \exp \left(i 2 k r^{\prime} \sin (\theta / 2) \cos \theta^{\prime}\right) r^{\prime} d r^{\prime} d \theta^{\prime}
\end{aligned}
$$

Here we have extended the limit of the integral in $r^{\prime}$ to $r^{\prime}=\infty$, assuming that the correlation function is small for offsets larger than the size of the heterogeneous zone. Since

$\int_{-\pi}^{\pi} e^{i x \cos \theta} d \theta=2 \pi J_{0}(x)$

[Hildebrand, 1962], we find

$$
\left(\frac{\Phi}{\Phi_{0}}\right)^{2}=\frac{k^{3} \sigma^{2} A}{|\mathbf{x}|} \int_{r=0}^{r=\infty} N\left(r^{\prime}\right) r^{\prime} J_{0}\left[2 k r^{\prime} \sin (\theta / 2)\right] d r^{\prime}
$$


The integral in equation (C6) is the two-dimensional Fourier transform $P\left(k_{r}\right)$ of $N(r)$ (see equation (6) in text), so that

$$
\left(\frac{\Phi}{\Phi_{0}}\right)^{2}=\frac{k^{3} \sigma^{2} A}{|\mathbf{x}|} P[2 k \sin (\theta / 2)]
$$

This formula states that for a seismic wave at wave number $k$, the energy scattered at angle $\theta$ is proportional to the fluctuation power spectrum of the medium at radial wave number $2 k \sin (\theta / 2)$. This is similar to the result of Malin and Phinney [1985] for three-dimensional media.

The scattering energy is now integrated over the scattering angle $\theta$ to determine the scattering attenuation $\left(Q^{-1}\right)$. Here we follow the procedure of $W u$ [1982] altered to the twodimensional case. For a plane wave propagating through a series of thin rectangles with thickness $a$ (in the propagation direction) and height $l$ (see $W u$ [1982] for geometry), the fractional energy lost by scattering in each rectangle is

$$
\frac{\Delta E}{E}=\frac{1}{l} \int_{b}\left(\frac{\Phi}{\Phi_{0}}\right)^{2} d S
$$

where $S$ is the arc length through which the scattered energy passes and $b$ describes the range of scattering angles. Since $Q^{-1}$ equals the energy loss per unit travel distance divided by $k$, we find

$$
Q^{-1}(k)=\frac{1}{\text { alk }} \frac{\Delta E}{E}
$$

Now $d S=r d \theta \approx|\mathbf{x}| d \theta$ and $A=a l$, so that substitution from equations (C7) and (C8) produces

$$
Q^{-1}(k)=2 k^{2} \sigma^{2} \int_{\theta_{\min }}^{\pi} P[2 k \sin (\theta / 2)] d \theta
$$

Here $\theta_{\min }$ and $\pi$ represent the range of scattering angles considered.

The frequency dependence of apparent attenuation in various random media can be evaluated from equation (C10), using the Fourier transforms listed in Table 1. For Gaussian media this yields

$$
Q^{-1}(k)=k^{2} \sigma^{2} a^{2} \int_{\theta_{\min }}^{\pi} e^{-k_{m^{2} a^{2} / 4}} d \theta
$$

and for exponential media

$$
Q^{-1}(k)=2 k^{2} \sigma^{2} a^{2} \int_{\theta_{\min }}^{\pi}\left(1+k_{m}{ }^{2} a^{2}\right)^{-3 / 2} d \theta
$$

where $k_{m}=2 k \sin (\theta / 2)$. In the case of a self-similar medium, inserting the right-hand side of equation (B12) into equation (C10) produces

$$
Q^{-1}(k)=0.58 k^{2} \sigma_{\text {ref }}{ }^{2} a^{2} \int_{\theta_{\min }}^{\pi}\left(1+k_{m}{ }^{2} a^{2}\right)^{-1} d \theta
$$

where $\sigma_{\text {ref }}$ is the standard deviation of the reference discrete medium $\left(a_{\text {ref }}=200 \mathrm{~m}\right.$ and $\left.h=20 \mathrm{~m}\right)$. The theoretical curves for apparent attenuation shown in Figures $16-18$ and 20 were derived by numerically integrating equations (C11)-(C13).

Acknowledgments. John Vidale developed and implemented a substantial portion of the finite difference codes used in this study. W. Menke, P. E. Malin, J. Boatwright, T. C. Hanks, D. J. Andrews, E. Cranswick, and an anonymous reviewer provided useful comments on the manuscript. We particularly thank one of the Associate Editors of JGR for suggestions which significantly improved the paper.
The research in this paper was carried out by the Jet Propulsion Laboratory and the Seismological Laboratory, California Institute of Technology, and was sponsored by the Sun Oil Company, Ametek Corporation, and the National Aeronautics and Space Adminstration. Contribution 4249 of the Division of Geological and Planetary Sciences, California Institute of Technology, Pasadena.

\section{REFERENCES}

Aki, K., Analysis of seismic coda of local earthquakes as scattered waves, J. Geophys. Res., 74, 615-631, 1969.

Aki, K., Scattering of $P$ waves under the Montana LASA, J. Geophys. Res., 78, 1334-1346, 1973.

Aki, K., Attenuation of shear waves in the lithosphere for frequencies from 0.05 to $25 \mathrm{~Hz}$, Phys. Earth Planet. Inter., 21, 50-60, 1980a.

Aki, K., Scattering and attenuation of shear waves in the lithosphere, J. Geophys. Res., 85, 6496-6504, $1980 b$.

Aki, K., and B. Chouet, Origin of coda waves: Source, attenuation, and scattering effects, J. Geophys. Res., 80, 3322-3342, 1975.

Aki, K., and P. G. Richards, Quantitative Seismology, W. H. Freeman, San Francisco, Calif., 1980.

Aki, K., A. Christofferson, and E. S. Husebye, Determination of the three-dimensional seismic structure of the lithosphere, J. Geophys. Res., 82, 277-296, 1977.

Alford, R. M., K. R. Kelly, and D. M. Boore, Accuracy of finite difference modeling of the acoustic wave equation, Geophysics, 39, 834-842, 1974.

Alterman, Z. S., and F. C. Karal, Jr., Propagation of elastic waves in layered media by finite-difference methods, Bull. Seismol. Soc. Am., $58,367-398,1968$.

Anderson, J. G., and S. E. Hough, A model for the shape of the Fourier amplitude spectrum of acceleration at high frequencies, Bull. Seismol. Soc. Am., 74, 1969-1993, 1984.

Andrews, D. J., A stochastic fault model, 1, Static case, J. Geophys. Res., 85, 3867-3877, 1980.

Berrill, J. B., A study of high-frequency strong ground motion from the San Fernando earthquake, Ph.D. thesis, Calif. Inst. of Technol., Pasadena, 1975.

Berteussen, K. A., Crustal structure and $P$-wave travel time anomalies at NORSAR, J. Geophys., 4I, 71-84, 1975.

Berteussen, K. A., E. S. Husebye, R. F. Mereu, and A. Rau, Quantitative assessment of the crust-upper mantle heterogeneities beneath the Gauribidanur seismic array in southern India, Earth Planet. Sci. Lett., 37, 326-332, 1975a.

Berteussen, K. A., A. Christofferson, E. S. Husebye, and A. Dahle, Wave scattering theory in analysis of $P$-wave anomalies at NORSAR and LASA, Geophys. J. R. Astron. Soc., 42, 403-417, $1975 b$.

Bouchon, M., The complete synthesis of seismic crustal phases at regional distances, J. Geophys. Res., 87, 1735-1741, 1982.

Chernov, L. A., Wave Propagation in a Random Medium, McGrawHill, New York, 1960.

Clayton, R., and B. Engquist, Absorbing boundary conditions for acoustic and elastic wave equations, Bull. Seismol. Soc. Am., 67, $1529-1540,1977$

Cranswick, E., R. Wetmiller, and J. Boatwright, High-frequency observations and source parameters of microearthquakes recorded at hard-rock sites, Bull. Seismol. Soc. Am., 75, 1535-1568, 1985.

Dainty, A. M., High-frequency acoustic backscattering and seismic attenuation, J. Geophys. Res., 89, 3172-3176, 1984.

Fletcher, J. B., Spectra from high-dynamic range digital recordings of Oroville, California aftershocks and their source parameters, Bull. Seismol. Soc. Am., 70, 735-755, 1980.

Fletcher, J. B., A comparison between the tectonic stress measured in situ and stress parameters from seismic waves at Monticello, South Carolina: A site of induced seismıcity, J. Geophys. Res., 87, 69316944, 1982.

Frankel, A., Precursors to a magnitude 4.8 earthquake in the Virgin Islands: Spatial clustering of small earthquakes, anomalous focal mechanisms, and earthquake doublets, Bull. Seismol. Soc. Am., 72, 1277-1294, 1982a.

Frankel, A., The effects of attenuation and site response on the spectra of microearthquakes in the northeastern Caribbean, Bull. Seismol. Soc. Am. 72, 1379-1402, $1982 b$.

Frankel, A., and R. W. Clayton, A finite difference simulation of wave propagation in two-dimensional random media, Bull. Seismol. Soc. Am., 74, 2167-2186, 1984.

Gao, L. S., L. C. Lee, N. N. Biswas, and K. Aki, Comparison of the 
effects between single and multiple scattering on coda waves for local earthquakes, Bull. Seismol. Soc. Am., 73, 377-390, 1983.

Geller, R. J., and C. S. Mueller, Four similar earthquakes in central California, Geophys. Res. Lett., 7, 821-824, 1980.

Hanks, T. C., $f_{\max }$, Proceedings of Workshop XVI, The Dynamic Characteristics of Faulting Inferred From Recordings of Strong Ground Motion, U.S. Geol. Surv. Open File Rep., 82-591, 405-436, 1982.

Haskell, N., Crustal reflection of plane $S H$ waves, J. Geophys. Res., 65 , $4147-4150,1960$.

Hearn, T. M., and R. W. Clayton, Lateral velocity variations in southern California, 1, Results for the upper crust from $P_{g}$ waves, Bull. Seismol. Soc. Am. in press, $1986 a$.

Hearn, T. M., and R. W. Clayton, Lateral velocity variations in southern California, 2, Results for the lower crust from $\boldsymbol{P}_{n}$ waves, Bull. Seismol. Soc. Am., in press, $1986 b$.

Hermann, R. B., $Q$ estimates using the coda of local earthquakes, Bull. Seismol. Soc. Am., 70, 447-468, 1980

Hildebrand, F. B., Advanced Calculus for Applications, Prentice-Hall, Englewood Cliffs, N. J., 1962.

Hudson, J., and J. R. Heritage, The use of the Born approximation in seismic scattering problems, Geophys. J. R. Astron. Soc., 66, 221240, 1981.

Humphreys, E., Studies of the crust-mantle system beneath southern California, Ph.D. thesis, Calif. Inst. of Technol., Pasadena, 1985.

Kelly, K. R., R. W. Ward, S. Treitel, and R. M. Alford, Synthetic seismograms: A finite difference approach, Geophysics, 41, 2-27, 1976.

Kraut, E., Fundamentals of Mathematical Physics, McGraw-Hill, New York, 1967.

Levander, A. R., and N. R. Hill, P-SV resonances in irregular lowvelocity surface layers, Bull. Seismol. Soc. Am., 75, 847-864, 1985.

Malin, P. E., and R. A. Phinney, On the relative scattering of $P$ and $S$ waves, Geophys. J. R. Astron. Soc., 80, 603-618, 1985.

Malin, P. E., and J. A. Waller, Preliminary results from vertical seismic profiling of Oroville microearthquake S-waves, Geophys. Res. Lett., 12, 137-140, 1985.

Mandelbrot, B. B., Fractals, W. H. Freeman, San Francisco, Calif., 1977.

Mandelbrot, B. B., and J. R. Wallis, Computer experiments with fractional Gaussian noises, Water Resour. Res., 5, 228-267, 1969.

McLaughlin, K. L., L. R. Johnson, and T. V. McEvilly, Twodimensional array measurements of near-source ground accelerations, Bull. Seismol. Soc. Am., 73, 349-376, 1983.

Menke, W., Asymptotic formulas for the apparent $Q$ of weakly scattering three-dimensional media, Bull. Seismol. Soc. Am., 74, 1079$1081,1984$.

Menke, W., and R. Chen, Numerical studies of the coda fall-off rate of multiply-scattered waves in randomly layered media, Bull. Seismol. Soc. Am., 74, 1605-1614, 1984.

Oberhettinger, F., Tables of Bessel Transforms, Springer-Verlag, New York, 1972.
Pechmann, J. C., and H. Kanamori, Waveforms and spectra of preshocks and aftershocks of the 1979 Imperial Valley, California, earthquake: Evidence for fault heterogeneity, J. Geophys. Res., 87, 10,579-10,597, 1982.

Pekeris, C. L., Note on the scattering of radiation in an inhomogenous medium, Phys. Rev., 7I, 268, 1947.

Powell, C. A., and A. S. Meltzer, Scattering of $P$-waves beneath SCARLET in southern California, Geophys. Res. Lett., 11, 481-484, 1984.

Rautian, T. G., and V. I. Khalturin, The use of the coda for determination of the earthquake source spectrum, Bull. Seismol. Soc. Am., 68, 923-948, 1978.

Richards, P. G., and W. Menke, The apparent attenuation of a scattering medium, Bull. Seismol. Soc. Am., 73, 1005-1022, 1983.

Ringdal, F., and E. S. Husebye, Application of arrays in the detection, location, and identification of seismic events, Bull. Seismol. Soc. Am., 72, S201-S224, 1982.

Roecker, S. W., B. Tucker, J. King, and D. Hatzfeld, Estimates of $Q$ in central Asia as a function of frequency and depth using the coda of locally recorded earthquakes, Bull. Seismol. Soc. Am., 72, 129-149, 1982.

Sato, H., Energy propagation including scattering effects single isotropic scattering approximation, J. Phys. Earth, 25, 27-41, 1977.

Singh, S. K., R. J. Apsel, J. Fried, and J. N. Brune, Spectral attenuation of $\mathrm{SH}$ waves along the Imperial fault, Bull. Seismol. Soc. Am. 72, 2003-2016, 1982.

Smith, S. W., J. E. Ehrenberg, and E. N. Hernandez, Analysis of the El Centro differential array for the 1979 Imperial Valley earthquake, Bull. Seismol. Soc. Am., 72, 237-258, 1982.

Spieth, M. A., Two detailed seismic studies in central California, part I, Earthquake clustering and crustal structure studies of the San Andreas fault near San Juan Bautista, part II, Seismic velocity structure along the Sierra foothills near Oroville, California, Ph.D. thesis, Staniord Univ., Stanford, Calif., 1981.

Tatarski, V. I., Wave Propagation in a Turbulent Medium, McGrawHill, New York, 1961.

Thurber, C. H., Earthquake locations and three-dimensional structure in the Coyote Lake area, central California, J. Geophys. Res., 88, 8226-8236, 1983.

$\mathrm{Wu}, \mathrm{R}$.-S., Attenuation of short period seismic waves due to scattering, Geophys. Res. Lett., 9, 9-12, 1982.

Wu, R.-S., and K. Aki, Scattering characteristics of elastic waves by an elastic heterogeneity, Geophysics, 50, 582-595, 1985.

R. W. Clayton, Seismological Laboratory, California Institute of Technology, Pasadena, CA 91125.

A. Frankel, U.S. Geological Survey, 345 Middlefield Road, MS/977, Menlo Park, CA 94025.

(Received August 18, 1985;

revised January 23, 1986;

accepted January 30,1986 .) 